\title{
Formalizing the Transition from Requirements Change to Design Change Using an Evolutionary Traceability Model
}

\author{
Lian Wen, David Tuffley, R.Geoff. Dromey \\ Software Quality Institute, Griffith University, Brisbane, Australia \\ \{1.wen, d.tuffley\}@griffith.edu.au
}

\begin{abstract}
The ideal outcome when responding to changes in the functional requirements of a system is that we can quickly determine (1) where to make the change, (2) how the change affects the architecture of the existing system, (3) which components of the system are affected by the change, and (4) what behavioral changes will need to be made to the components (and their interfaces) that are affected by the change of requirements. If these facts are known, the impact of the change is entirely understood and therefore manageable. Moreover, a system is likely to undergo multiple changes over the course of its service life, so there is also a need to make a comprehensive record of these changes thus preserving the integrity of the system and potentially extending its service life.
\end{abstract}

To this worthy end, a traceability model using behavior trees as a formal notation to represent functional requirements is proposed. This will address the issues cited above, revealing change impacts on different types of design constructs (documents) caused by the changes to the requirements. The proposed model introduces the concept of evolutionary design documents that record the change history of the designs. From these documents, any version of a design document as well as the difference between any two versions can be reviewed, thus affording the desirable condition of full traceability.

An important advantage of this model is that the major part of the procedure to generate these evolutionary design documents can be supported by automated tools making the method accessible for use in large-scale software and systems development projects.

Keywords: software change, behavior tree, behavior engineering, traceability

analysis, software evolution, requirements engineering.

\section{Introduction}

It is in the nature of software systems that they are subject to ongoing change [40]. According to Somerville [67](page 235) in order to keep a software system useful, it needs to be changed after its deployment. Among different types of software changes, the type of change that has the most impact is software enhancive change [10] (also called "Perfective" [33], "Evaluative" [31], or "New Requirements" [35]). The intensity of the impact of this on-going change is often magnified due to the introduction of new requirements that modify or remove existing requirements.

When new requirements are introduced it is essential to do three things to ensure integrity. These are to; (a) map the changes from the problem domain to the solution domain [77], (b) determine the change impact [84], and (c) maintain the traceability [43].

Within the lifetime of a software system, multiple releases and many development cycles are expected [57]. To manage this ongoing change of a software system, it is advantageous to have a utility that can keep the horizontal traceability between the user requirements, the design documents and the source code, while also maintaining vertical traceability (Fig 1) along the evolutionary track of the software system. Lindvall defines vertical traceability as that between different software artifacts of the same type within the same version, e.g. the traceability between two requirements in a software system [43]. In contrast, this paper defines vertical traceability as 
that of the same design artifact in different versions, a significant distinction from Lindvall's definition. There will be evolutionary changes from one version to the next. It is possible, even likely those significant differences will exist between the earliest and the latest versions. This would suggest a compelling case for the development of a method to manage this vertical traceability.

There are several established techniques that support software change impact analysis and manage traceability between requirements specification and

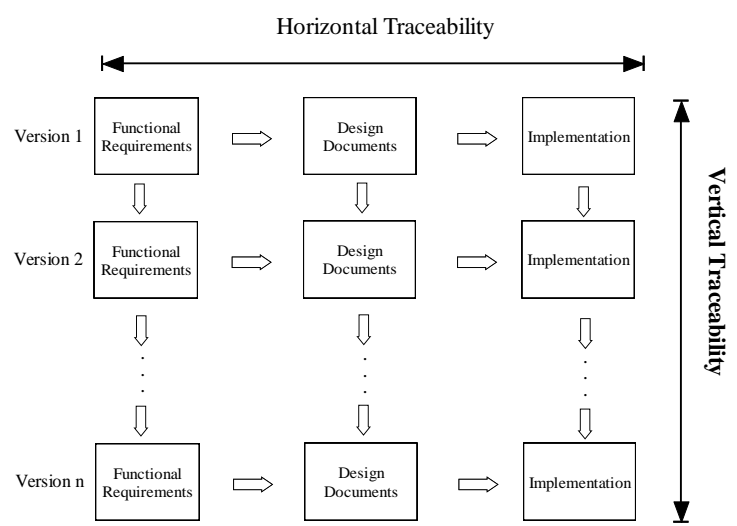

Fig. 1. Horizontal and vertical traceability. design documents. These include program slicing [23] at the code level [84] and/or at the design level [68], certain environments based on hypertext systems [13][72], such as DIF [24], and also environments based on the use of a relational database such as SODOS [32]. A limitation of these latter environments is that they require users to engage in the time consuming manual creation of linkages between different documents.

In recent times a number of new techniques have been developed to support change impact analysis and manage the traceability and dependency of a software system. Architectural slicing and chopping have been proposed to support change impact analysis at the architectural level [84] and to study the evolution of aspect-oriented software [85]. Architectural-level dependence has also been studied [70] using the architecture description languages (ADL) Rapide [44]. The softgoal interdependency graph (SIG) has been used to model non-functional requirements (NFR) [14] and algorithms have been explored to compare different meta-models (graphs) for reviewing the evolution of general models [2][21][37] and/or UML diagrams [61].

On a different level, the B abstract machine [60] claims to be able to automatically produce a traceability matrix from requirements to test cases [7]. While these various techniques make useful contributions to the discipline, few of them provide an efficient method to (a) handle vertical traceability by comparing multiple versions of a software system, (b) visualize in a graphical form the evolution of the architecture and individual components, and (c) trace the design evolution back to the requirement evolution.

Therefore, in this paper, a traceability model, which is an extension of previous work [77] is proposed.

The traceability model is implemented at the planning phase in the mini-cycle of software change [75][55]. In requirement engineering (RE), the corresponding activities at that phase involve modeling (or behavior modeling [81]) and analyzing requirements [51]. The purpose of these activities is to understand the (new) requirements and then to change or construct a design to satisfy them; in other words to map the changes from the requirements to the design. There is a wide range of informal and formal methods for doing this modeling [51]. The informal methods usually provide rich representations [54] that non-technical stakeholders find understandable, but they are often difficult to process automatically. Meanwhile, methods based on formal representations, such as Z [58] or B [60], are amenable to automated analysis but are difficult to construct and understand by non-technical stakeholders. In contrast, the proposed traceability model uses Behavior Trees (BT) [15] as the notation. BT is easy to understand because it strictly retains the behavior vocabulary of the original requirements while using a simple and expressive graphic notation, while also having a formal semantic structure [12] so that automated analysis such as model checking [82] and simulation [80] can be performed.

The traceability model introduces a concept called evolutionary design diagrams, which displays designs of multiple versions in one graph with clear visual hints to indicate which part of the designs belong to which version. Through evolutionary design diagrams, designers can grasp the evolution of different design artifacts. These evolutionary design diagrams also provide the traceability from the designs back to the requirements, thus helping people to understand the rationale of the design evolution. 
There are four different types of evolutionary diagrams that can be obtained from the traceability model. The first is called an evolutionary design behavior tree (EvDBT), which captures the evolution of the behavior of the entire system. The second is called an evolutionary component behavior tree (EvCBT), which captures the evolution of the behavior of a specific component. The third is called an evolutionary component interface diagram (EvCID), which shows the evolution of the interfaces of one component. The last one is known as an evolutionary component integration network (EvCIN), which represents the component architecture (or component dependency relationship) of the system. Details of these diagrams will be introduced in Section 3.

The EvDBT is generated by applying a top-down tree merging algorithm based on unique names (discussed in Section 3) on several behavior trees (DBT). The remaining three evolutionary design diagrams are directly projected from the EvDBT.

The proposed traceability model provides a straightforward approach to map the change and evolution in functional requirements to the change and evolution of design artifacts, including the component architecture, component behavior and component interface. The strength of this model is that it not only maintains the horizontal traceability of information between the requirement specifications and software designs, but also maintains the vertical evolutionary information (Fig 1) of the same design documents across different versions.

An important advantage of the proposed model is that the evolutionary information and the traceability information displayed in the evolutionary documents, allow questions such as what is the current design, how did it come to be this and when did it become this to be answered. Based on these answers, the design rationale question [9] of why the current design is like this may also be answered. This knowledge might help the system maintainers to implement further changes with less impact of the system [76][80] and eventually extend the life of the software system. However further research and experiments will be required to verify this point.

This paper also presents two case studies; the microwave oven [63] and the satellite control system. This latter is a relatively large system with 14 pages of requirements [66] which demonstrates the ability of the proposed method to effectively deal with large and/or complex systems. The case studies help the reader to understand the nature and operation of the traceability model.

The remaining sections of this paper are organized as follows: the Behavior Engineering (BE) approach is briefly introduced in section 2 . This section is useful for people who are not familiar with this technique, which is essential to understand the proposed traceability method described in detail in section 3. Readers who are already familiar with the BE approach can proceed directly to section 3. Section 4 outlines the two case studies to demonstrate the practicability and efficacy of the traceability model. Finally, we provide a conclusion and a discussion of future work.

\section{Behavior Engineering (BE)}

Behavior Engineering is a systematic procedure for constructing an integrated, formally-expressed specification from which a design is then derived, based on a set of functional requirements [4][17][20]. In contrast to conventional software engineering, which builds a software system that hopefully satisfies a set of requirements, the BE approach derives a software system out of its requirement set.

Traditional software engineering provides no formal procedure to create a design from the functional requirements. While some design activities are suggested [57][67], there is no welldefined process to convert the requirements into a design, or put another way, to derive the design from the requirements. This shortcoming makes it difficult to ascertain the suitability or otherwise of a design based on objective criteria. There is also the matter of a lack of repeatability.

To overcome these problems, the BE approaches the problem from a fresh perspective. Rather than creating a design that appears to satisfy a set of requirements, it first constructs a specification, and then derives the design from the requirements. This approach has three major steps. 
- The first step rigorously translates (in terms of intention-preserving and vocabularypreserving criteria) each individual functional requirement into a corresponding requirements behavior tree $(\mathrm{RBT})$.

- $\quad$ The second step integrates all the RBTs a Design Behavior Tree (DBT) .

- The third step generates design diagrams from the DBT. These diagrams reveal the component architecture, the logic and behavior within each component and the interfaces of each component.

It should be noted that this section provides a relatively informal introduction to Behavior Engineering. A more comprehensive description of the approach is given elsewhere [15][17]. There has also been a range of other work published on Behavior Trees. Gonzalez-Perez, et al has defined a meta-model for a core part of the notation [25]. Winter, Grunske and Colvin have shown Behavior Trees can be translated into other formal specification languages such as CSP [82], SAL [26] and UPPAAL [29], so that model checking and simulation can be performed. At the same time, an EBNF styled textual semantic language (BTSL) has been developed [12]. Behavior trees have also been used to detect requirements defects at an early stage [16]. Modeling of nonfunctional requirements e.g., safety requirements and the security requirements in an embedded system [83] has been studied and an automatic process for Failure Modes and Effects Analysis (FMEA) [26] has also been developed. Other work includes the verification of process reference models [73], software change impact analysis [77], architecture normalization [76], large-scale system case studies [18], direct $\mathrm{C}++$ and Java code generation and software tools and environments [64][42][78]. Myers et al [48] have made the important step of interfacing Behavior Trees with Modelica, an industrial strength technology which is an open standard for system architecture and mathematical modeling. Modelica provides powerful support for modeling and simulating applications composed of complex physical systems.

\subsection{Behavior Trees}

A Behavior Tree is a formal, tree-like graphical form that represents the behavior of individual or networks of entities which realize or change states, create and break relations, make decisions, respond-to and/or cause events, and interact by exchanging information and/or passing control. Conventions for, and use of the more powerful executable, extended relational notation are outside of the scope of this paper.

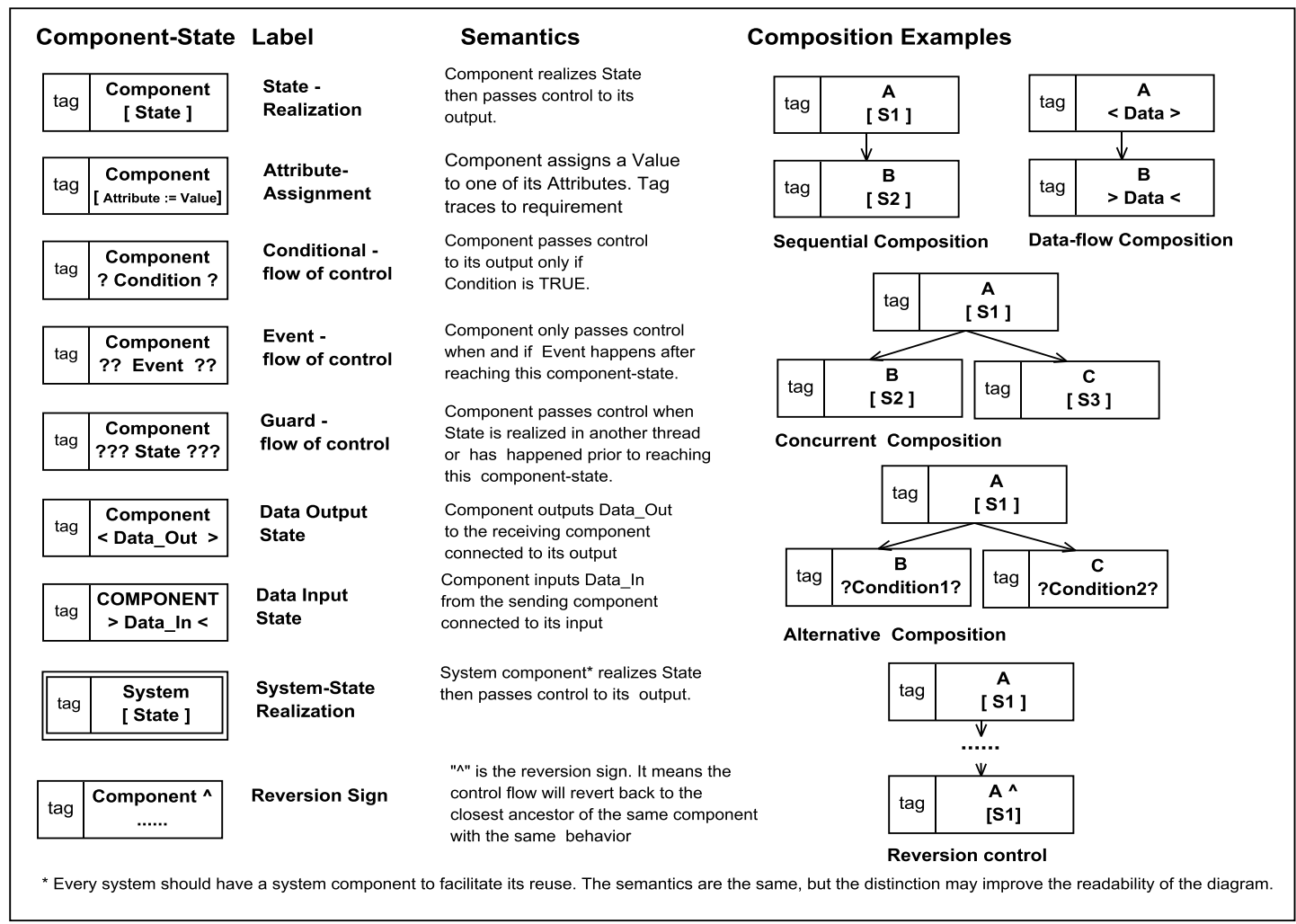


Fig. 2 indicates the core elements of Behavior Tree notation.

Behavior is expressed in terms of components realizing states. The description is augmented by the graphical conventions found in programming languages that are used to describe actions, composition, events, control-flow, data-flow, and threads. Behavior trees accurately capture behavior expressed in the natural language representation of functional requirements. They use the vocabulary of the natural language requirements but employ graphical forms for behavior composition which effectively eliminates the risk of ambiguity. In this way, Behavior Trees create a direct and traceable link between what is expressed in the natural language representation and its formal specification.

The rationale of the notation is that behavior is always associated with some component. Component-states representing nodes of behavior are composed sequentially and/or concurrently to construct a formal behavior tree that effectively represents the behavior expressed in natural language requirements.

In the BE approach, a component is initially defined as a design abstraction without an associated implementation specification. However, the lack of a component implementation specification does not make the meaning of a behavior tree ambiguous. Because a Behavior Tree has a formal semantics [12], its meaning is precisely defined and can be model checked [82][83] and simulated [78] using our existing tool environment.

A Behavior Tree therefore specifies state changes in components, how data is passed between components and how threads interact. In the following sub-sections, we will use simple examples to illustrate the major steps of the BE approach.

\subsection{Requirements Translation}

The first formal step in the development of Behavior Tree notation is requirements translation, the purpose of which is to translate each natural language functional requirement, one at a time, into a Requirement Behavior Tree (RBT). For example, the sentence "when the door is open the light turns on" is translated to the behavior tree seen in Fig. 3.

From Fig. 3, we can see the behavior tree includes two components "DOOR" and "LIGHT"; the event of "DOOR" "Open" will cause the behavior of "LIGHT" to realize the "On" state, which matches the original sentence.

Now let us consider a more complex example, that of a microwave oven system. This system was introduced by Shlaer and Mellor [63]. It has been used to explain the BE approach [17], and to discuss our traceability model [77], as well as the process of architecture normalization [76].

The original microwave oven case study included seven stated functional requirements:

- R1: There is a single control button available for the user of the oven. If the oven is idle with the door closed and you push the button, the oven will start cooking (that is, energize the power-tube for one minute).

- R2: If the button is pushed while the oven is cooking it will cause the oven to cook for an extra minute.

- R3: Pushing the button when the door is open has no effect (because it is disabled).

- R4: Whenever the oven is cooking or the door is open the light in the oven will be on.

- R5: Opening the door stops the cooking.

- R6: Closing the door turns off the light. This is the normal idle state, prior to cooking when the user has placed food in the oven.

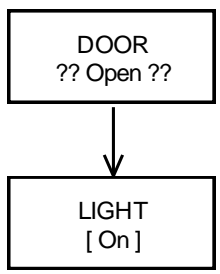

- R7: If the oven times-out the light and the power-tube are turned off and then a beeper emits a sound to indicate that the cooking is finished

Fig. 3. A behavior tree translated from the requirement, DOOR and LIGHT ARE two components. The tree means the event of DOOR Open will cause LIGHT to realize the state of On. 
The translation for requirement 7 (R7) is shown in Fig. 4. From Fig. 4, we can see that, initially, the OVEN is in the "Cooking" state. When the OVEN times-out, the LIGHT is off, POWER-TUBE is off, BEEPER sounds etc. The "+" sign (and also the yellow color) in the root state "OVEN [Cooking]" indicates these states are only implied in the original requirement. The behavior trees translated for the complete set of requirements can be found elsewhere [17].

\subsection{Requirements}

\section{Integration}

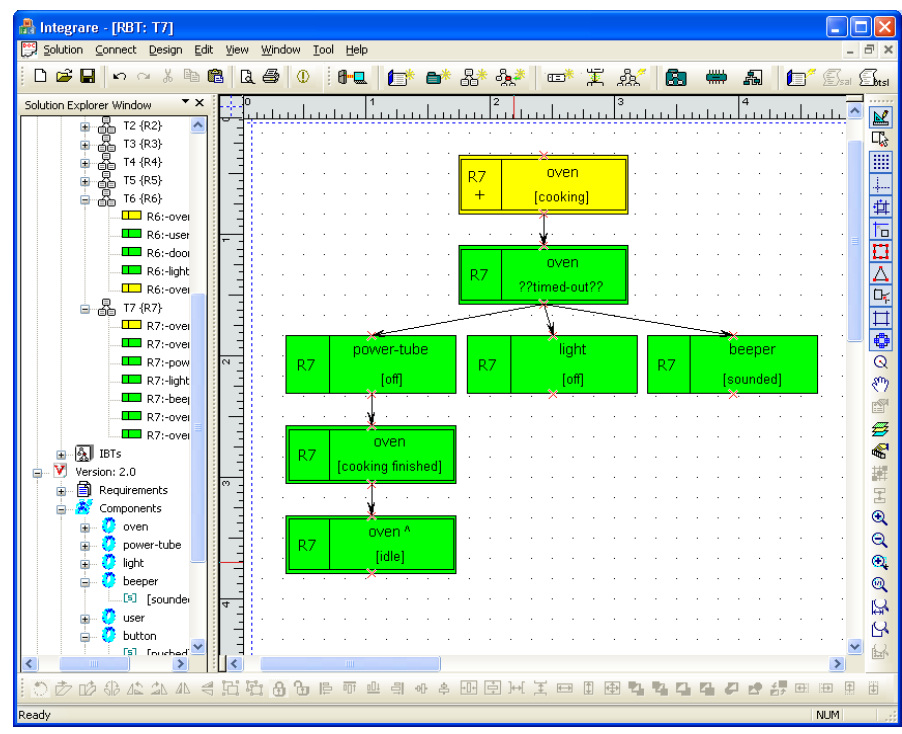

Fig. 4. Behavior tree for requirement R7.

After all of the requirements have been translated into RBTs, these are systematically and incrementally integrated into a design behavior tree (DBT), which covers all the requirements (as discussed earlier, we assume any defects discovered during this process are corrected along the way). A formal description of the integration rules requires the Precondition Axiom and the Interaction Axiom [15]. RBT integration is discussed below.

Each behavior tree must have a root node which serves as the foundational precondition for the behavior described by the rest of the behavior tree. If the root node of a tree appears somewhere in another behavior tree, it means the behavior of the second tree may satisfy the precondition of the

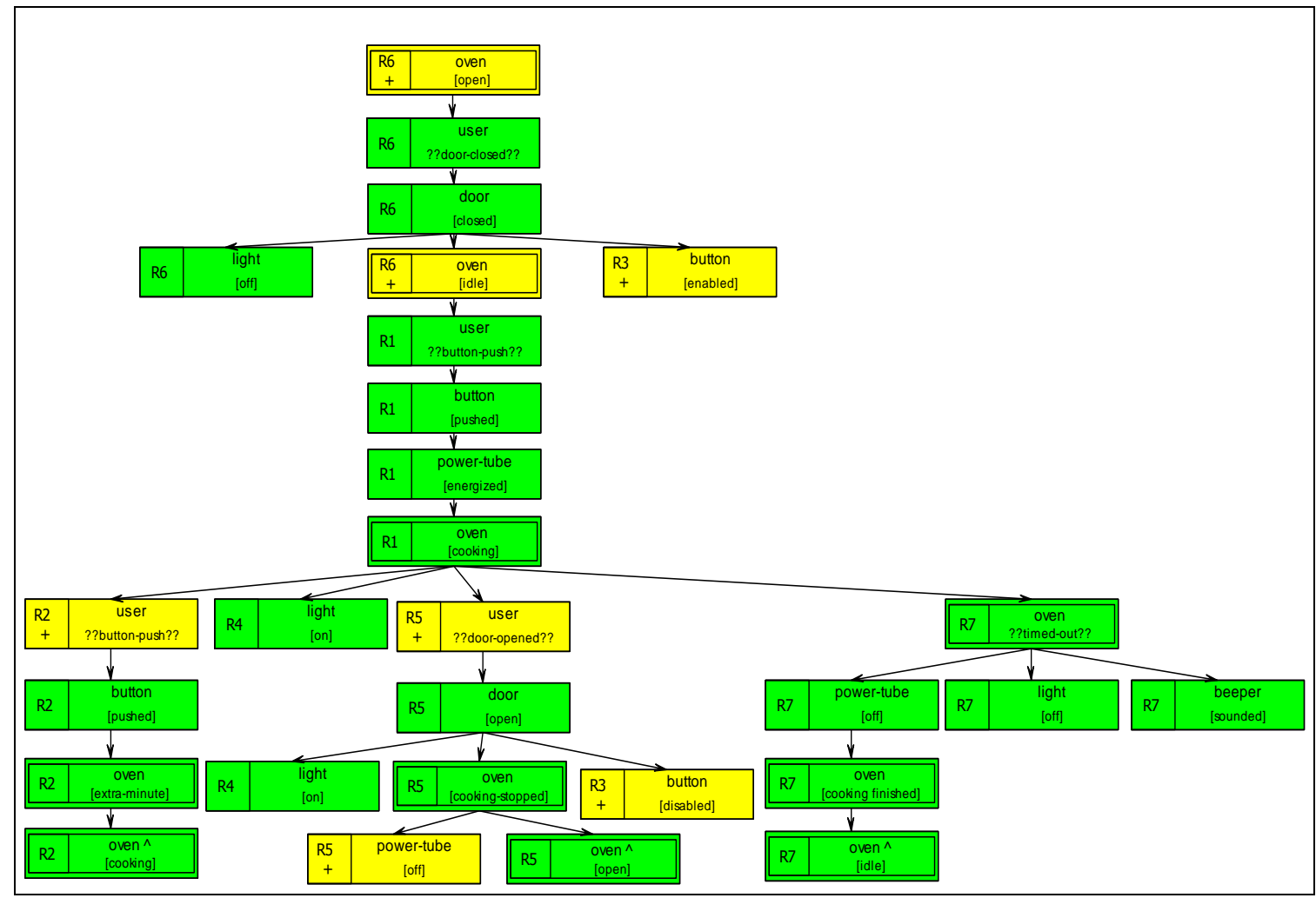

Fig. 5. The DBT for the Microwave Oven System. 
first tree. When this condition is satisfied the first tree can be integrated with the second tree. Using this behavior-tree grafting process, a complete design is constructed incrementally by integrating RBTs and/or DBTs pairwise until we are left with a single final DBT.

This is the ideal for design construction that is realizable when all requirements are consistent, complete, composable and do not contain redundancies.

A DBT shows all of the behaviors and all of the flows of control and data, modeled as component-state interactions without any of the functionality needed to realize the various states that individual components may assume. It embodies several emergent properties of a design, including (a) the component-architecture of a system, (b) the behaviors of each of the components, and (c) the interfaces of each of the components in the system. These properties are visualized by three design documents: a component integration network (CIN), a set of component behavior trees (CBTs), and a set of component interface diagrams (CID), which are introduced in the following subsections.

\section{4 Architecture Transformation}

For software systems the software architecture is a critical issue. According to Bass, "The software architecture of a program or computing system is the structure or structures of the system, which comprise software elements, the externally visible properties of those elements, and the relationships among them" [3]. In the BE approach, a component integration network (CIN) is developed which shows the integration (or dependency) relationships between all of the components in a software system. This provides an indicative view of the software architecture which we call component architecture.

In the Design Behavior Tree (DBT) representation, a given component may appear in different parts of the tree with different behaviors (e.g., the oven component may have the behavior [Idle] in one part of the tree and the behavior [cooking] in another part). We can convert a DBT to a component-based design in which each distinct component is represented only once. Similarly, the integration relationship between an ordered pair of components will also be represented only once. For example, there is more than one instance in Fig. 5 where the behavior of "oven" will determine the behavior of "user", but the integration relationship from "oven" to "user" will only be represented once in the architectural design. A simple algorithmic process may be employed to accomplish this transformation from a tree into a network. Informally, the process starts at the root of the Design Behavior Tree and travels downwards through all the child nodes (it is insignificant whether we use the depth first approach or breadth first approach). Whenever a new component is reached during the traversal process, that component will be drawn in the CIN. Similarly, if a new connection between two different components is discovered, that connection will also be drawn in the CIN. Generally, a connection from component A to component B is treated as a different connection from component B to component A. Once every node in the DBT has been processed, the corresponding CIN is completed. The CIN that is created from the DBT in Fig. 5 is shown in Fig. 6.

Comparing a CIN with an architecture presented in other architecture description languages (ADL) such as Rapide [44], Wright [49] and UniCon [62], people may argue that the information shown in a CIN is too simple. The meaning of an arrowed connection in a CIN is not clear; is it a data flow, a control flow, a connector defined in Wright [49], a channel or a method call? Our answer is that in the $\mathrm{BE}$ approach, all the information shown

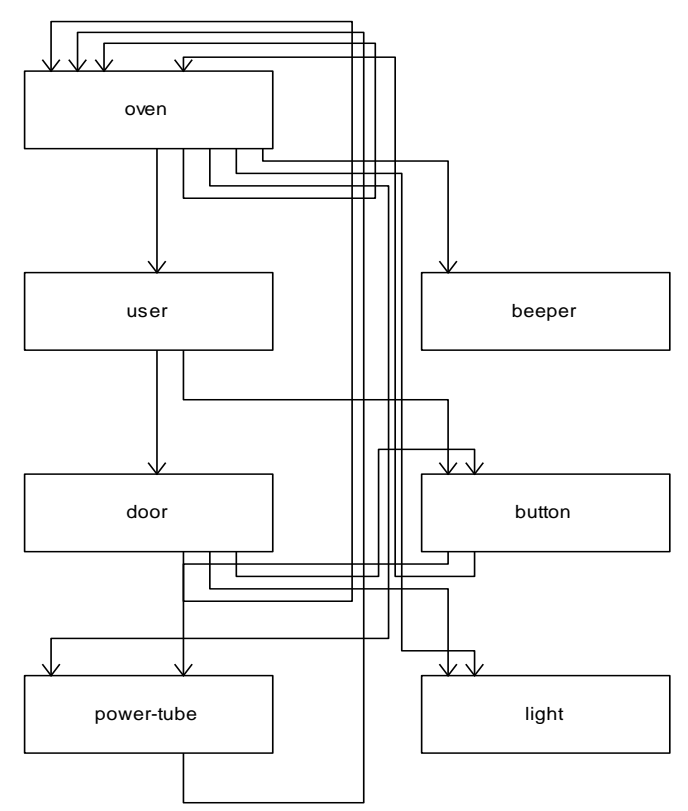

Fig. 6. The component integration network $(\mathrm{CIN})$ of the microwave oven system. 
corresponding Design Behavior Tree. If the DBT models a system abstractly, a component and a connection between two components are kept in an abstract form in the CIN as well. In this situation, if there is a connection between two components, what we can say is that the two components are integrated in the system and there is a dependency relationship between them. If a DBT provides more information about how the control flows from one component to another component, this information will also be captured in the CIN. In this situation, we may even have a CIN with two or more different connections from one component to another. For example, a component may communicate with another component through both shared memory and a pipe for different purposes. However, a CIN, even in the most abstract form, still provides information for dependency and change impact analysis.

The CIN provides a component integration network whereas the DBT provides a component integration specification. Earlier we pointed out that a DBT could be viewed as a set of interleaved component behavior trees. If we project out each component's behavior tree then design and implement the component so it encapsulates its CBT, we can then execute the DBT to implement the system by making appropriate state-parameterized calls to each component as each node in the DBT is encountered.

\section{5 Component Behavior Projection}

In the design behavior tree, the behavior of individual components tends to be dispersed throughout the tree. To implement components that can be embedded in, and operate within, the derived CIN, it is necessary to "concentrate" each component's behavior. We can achieve this by systematically projecting each component's behavior tree (CBT) from the DBT. We do this by simply ignoring the behaviors of all components other than the one we are currently projecting and taking into account any branching contexts. The resulting connected "skeleton" behavior tree for a particular component defines the behavior of the component that we will need to implement and encapsulate in the final component-based implementation. In some cases the projected behavior for a component contains redundancy that can be removed when the component's behavior is designed.

To illustrate the effect and significance of component behavior projection we show the projection of the "oven" component in Fig. 7. Component behavior projection is a key design step in the solution domain that needs to be done for each component in the design behavior tree.

A component behavior tree shows the behavior, the functional capacity and the logic associated with the functions of a component. For example, from Fig. 7, we know that the "oven" component initially is in the state of "[open]", then in the state of "[idle]" and then "[cooking]". When it is in the state of "cooking", the state can be changed to "[cooking-stopped]", "[extraminute]", or "??timed-out??"; which state the component will realize is determined by the system. A CBT shows all the possible state transform paths of a component in a tree-like form. Functionally, it is like a state transition diagram, but it is a tree rather than a network. Therefore, it is simpler in structure. An important issue is that a CBT is not drawn based on the intuition of the designers but based on the integrated behaviors described in the functional requirements. Suppose for example, a system requires a component "STACK". What is the state diagram of the "STACK", and what functions should it provide? Based on common knowledge, we know that a "STACK" must provide a "push" function and a "pop" function; we also know that a "pop" function can only be called after a "push" function. However, we do not know whether the "STACK" requires a "clear" function or "check capacity" function. These questions are not easy to answer. In the BE approach, because a CBT is a mapping from the design behavior tree, all the required functions as well

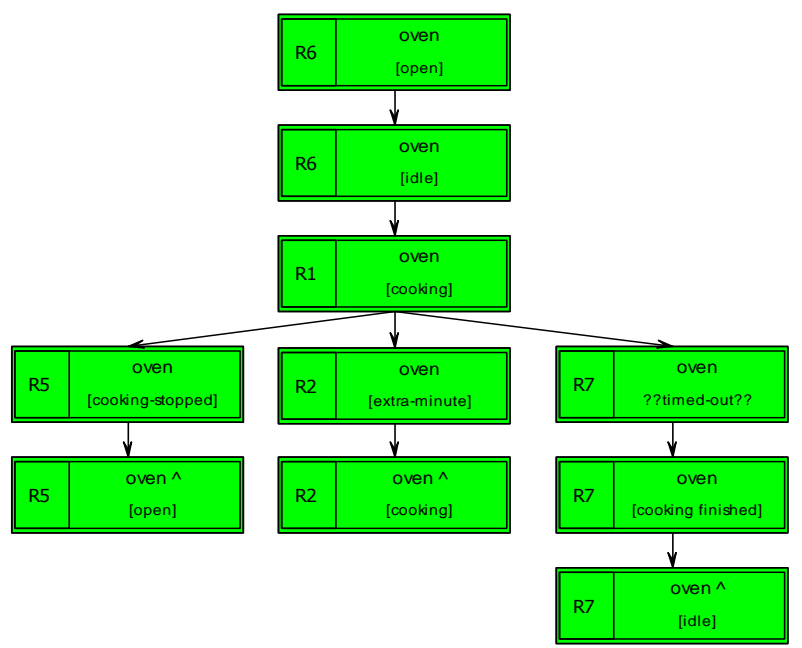

Fig. 7. The component behavior tree (CBT) of the "oven" 
as the order and logic of those functions are retrieved from system's behavior modeling of the requirements which provides the necessary context. This ensures there is no redundancy or missed functions unless there are defects in the functional requirements.

\section{6 Component}

\section{Interface Diagram}

A component interface diagram (CID) shows what other components the component interfaces with. A CID can be directly projected from the DBT. The first step to project a component's CID is to highlight all the nodes in the DBT of the given component, and

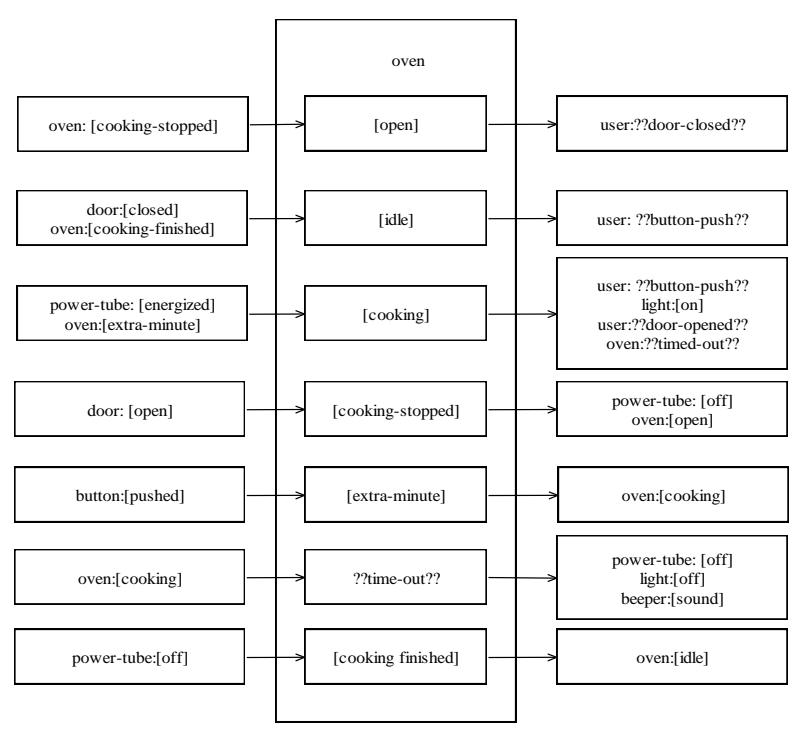

Fig. 8. The component interface diagram (CID) of the "oven"

then select all the links-to or links-from these nodes. From these links we can trace the components on the other end of these links. This yields the "input" components and "output" components of any given component. Fig. 8 shows the CID of the "oven" component projected from the DBT in Fig. 5. While a CBT shows what the internal functions and the logic of a component are, a CID shows how this component can be integrated with the environment and other components. Together, the CBT and CID act as a blueprint for the implementation of a component.

\subsection{Traceability Between Models}

The previous subsections briefly cover the main concepts and major steps of the BE approach. This subsection considers the different diagrams (models) of the BE approach, and also shows the traceability among the various diagrams (see Fig. 9).

From these diagrams, different platform-specific models and functional level designs can be subsequently created, but that is beyond the scope of this paper.

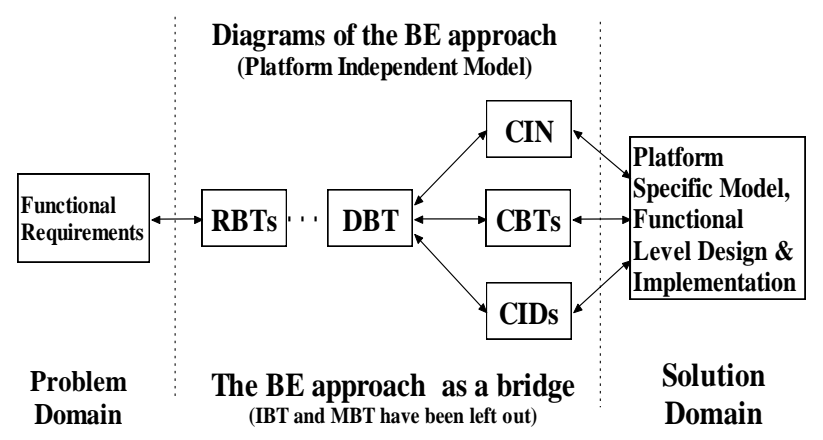

Fig. 9. The traceability in BE approach.

\section{From Requirements Change to Design Change}

\section{1 The Traceability Model}

Here we investigate construction of a traceability model, which can handle multiple changes over time. This overcomes the limitations of our earlier work for handling software changes [77], and is more practical because most software systems change many times during their life-time [47]. The essential idea of the model is illustrated in Fig. 10. 
This model addresses the problem of formalizing the impact of changes and evolution on the design. The output of the method is a set of evolutionary design diagrams which show the evolution of the design driven by the evolution of the requirements. More specifically, the evolutionary design diagrams not only show all the merged designs, but also show the similarity and difference among

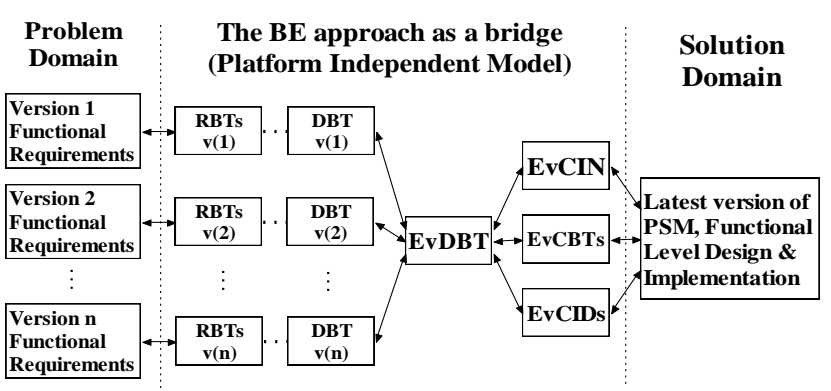

Fig. 10. The traceability model. those designs.

To understand the formalization of design evolution, suppose that we have a software system with several different versions of functional requirements. For each version of software requirements, a corresponding design is constructed using the BE process. The problem is how we could trace the design evolution back to the requirements evolution. Our approach, illustrated in Fig. 10, has three major steps:

1. Translate each set of the functional requirements into a corresponding set of RBTs, and then integrate each set of RBTs to produce the corresponding IBT which is refined into a DBT of that version.

2. Compare these DBTs by merging them together to produce an Evolutionary Design Behavior Tree (EvDBT). The EvDBT not only covers the complete information of all the designs, but it also covers the information of the similarity and difference among those designs, which shows the design evolution caused by the requirement evolution.

3. From the EvDBT, other evolutionary design documents including an EvCIN, EvCBTs and EvCIDs can be derived.

The overall process is similar to the original BE process, but it brings in two important concepts: the first concept is to compare several design behavior trees by merging them into one evolutionary design behavior tree; the process will be introduced in the following subsections. The second concept is an evolutionary design document. The difference between an evolutionary design document and a normal design document is that the former highlights the evolutionary information of the design.

Here we emphasize two points. Firstly, if the different versions a software system have been designed using the $\mathrm{BE}$ approach, then generating an evolutionary design behavior tree is simplified, because previous versions of the RBTs and DBTs are available and can therefore be used directly. Secondly, in order to generate an evolutionary design behavior tree, the designers do not need to merge the DBTs of all the existing versions. They need only select the versions they are interested in. Because the merging can be fully automated by software tools (the tools will be introduced in later sections), they can easily generate the evolutionary design behavior trees of the selected combination of versions.

As an evolutionary design document shows the evolutionary history of the design, which can be traced back to the evolution of the functional requirements. This feature will help the designers and developers understand large systems better and eventually reduce the cost to maintain those systems.

The process for deriving other evolutionary design diagrams from an EvDBT is similar to the process of projecting different design diagrams from a DBT; except it also maintains the version information. Details of the projection rules are discussed in the following subsection.

\section{2 The Tree Merging Algorithm and Projection Rules}

The process to merge different design behavior trees and generate an evolutionary design behavior tree is a core part of the traceability model. In this subsection, we will use a simple example to describe the algorithm for this process in an intuitive way. A formal definition of the algorithm is given in the next subsection. 
The tree merging algorithm, which is an extension of a previous version [77], is a top-down tree matching algorithm based on unique labeling. Compared with the previous version and many other algorithms used to merge software design diagrams [37][21], this merging algorithm has following features:

- While many other algorithms are designed to compare and merge two graphs, this algorithm can merge any number of trees simultaneously in parallel.

- Algorithms that merge more than two graphs can require user input to resolve contradictions in different versions before the merged view can be produced. The tree merging algorithm proposed in this paper requires no such user input or conflict resolution before generating the merged view.

- While many merge algorithms are designed for UML diagrams [61] or general graphs [37], few of them are designed specifically for behavior trees.

In the BE approach, a behavior tree is a collection of atomic items that are arranged in a treeformed graph. An atomic item can be either a node or a link. In a DBT, each atomic item has associated with it a number of different types of tags. For example, a requirement tag (R1, R2 and $\mathrm{R} 3$ in the microwave oven example) and also a version tag. In this section, we mainly focus on version tags.

Let $T_{1}, T_{2}$ and $T_{3}$ shown in Fig. 11, be the three DBTs of version $v_{1}, v_{2}$ and $v_{3}$ correspondingly. The version tag is not only associated with the tree, but it is also attached to every atomic item in that tree. Because in an EvDBT tree, an atomic item is usually associated with more than one version, a version tag is a set of versions.

To merge the three trees and generate the evolutionary design behavior tree, we use the following procedures:

1. Start the comparison with the root nodes (in this example, node A). Because node A exists in all the three trees as the root node, it is created in the evolutionary design behavior tree as a node $\mathrm{A}$ with the set $\left\{v_{1}, v_{2}, v_{3}\right\}$ as its version tag.

2. Find the child-node set for every compared node. (In this example, the child-node set of $\mathrm{A}$ from $T_{1}$ is $\{\mathrm{B}, \mathrm{C}\}$, the child-node set of $\mathrm{A}$ from $T_{2}$ is $\{\mathrm{G}, \mathrm{C}\}$, and child-node set of $\mathrm{A}$ from $T_{3}$ is also $\{\mathrm{G}, \mathrm{C}\}$.

3. Compare the child-node sets from step 2. If a node only exists in one of the child-node sets, this node will be created in the evolutionary tree with the same version tag as the original node, and all its sub trees will also be cloned with the same version tag. In this example, the node B from $T_{1}$ satisfies this criterion, so node B and its child node D are created in the EvDBT with version tag as $\left\{v_{1}\right\}$.

4. If a node existed in the childnode sets of compared nodes from more than one DBT, those nodes will be merged to generate a new node in the EvDBT with the version tag as the union of the version tag of all the merged nodes. In our example, node $\mathrm{G}$ in $T_{2}$ and $T_{3}$ is merged to generate a node $\mathrm{G}$ in the EvDBT with the version tag as $\left\{v_{2}, v_{3}\right\}$. Similarly, node $\mathrm{C}$ exists in the child-node sets from all the three trees, so a node $\mathrm{C}$ will be merged in the EvDBT with node set $\left\{v_{1}, v_{2}\right.$, $\left.v_{3}\right\}$.

5. If more than one node is: merged, these merged nodes will be the new compared nodes and the process will go back recursively to step 2 .

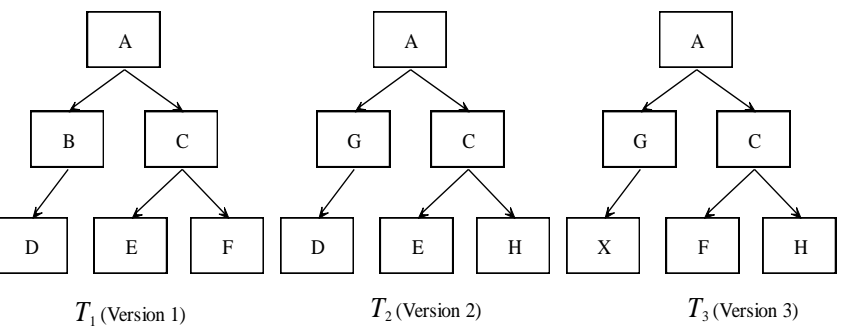

Fig. 11. The three DBTs $T_{1}, T_{2}$ and $T_{3}$.

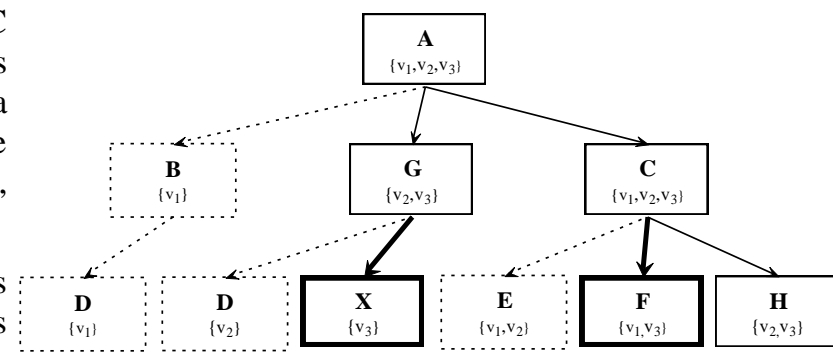

Fig. 12. The edited design behavior tree $T_{\mathrm{e}}$. 
Following the procedure above, we have the merged EvDBT $T_{\mathrm{e}}$ shown in Fig. 12. The version tag of each node is displayed in the node. An atomic item (here we mean a node or a link) with the version tag including both the latest version $\left(v_{3}\right)$ and the second latest version $\left(v_{2}\right)$ is printed in the normal style. An atomic item with the version tag including the latest version but not the second latest version is printed in bold, which means the item is newly added in the latest version. An atomic item with the version tag not including the latest version tag is printed using a dotted line, which means that part has been removed from the latest version. The same notation is also used in the case study discussed in the next section, but of course, for different purposes. A designer may select different display schemes to satisfy his/her usage.

Several points are worth noting. The first is about the version tag of links. In a tree, each link has two connected nodes, a parent node and a child node. In an evolutionary design behavior tree, the associated version tag for a link is defined as that of its child node. The reason for this is that during the tree merging process, each link and its associated child node can be treated as one entity.

Secondly, the final evolutionary design behavior tree is derived from the merging of a number of design behavior trees. The order in which the trees have been merged is not an issue, since the process follows the commutative law. A formal proof of this point is given in the next subsection. This feature is important, because it avoids the troublesome conflicts caused by asynchronous changes, where different designers and developers modify the system separately and cause inconsistency problems [71].

The third point concerns the naming of the node. This is determined by the name and behavior of the associated component. If the name of a component in a particular version has been changed, a mapping function is required to identify the matching nodes.

The final point concerns the movement of nodes. In the merging algorithm, if a node has been moved to different locations in other versions, the merged tree will have multiple copies of that node. For example, in Fig. 12, there are two nodes of $D$, caused by the movement of the node in version 1 and version 2 . A solution to this problem requires the projection of several other evolutionary design diagrams that are discussed below:

As discussed before, the process of projecting evolutionary design diagrams from an EvDBT involves the EvDBT being decomposed into its constituent atomic items which is either a node or a link. Each item is associated with a set of versions. When an evolutionary design diagram is projected from the EvDBT, any atomic item in the new diagram is able to be traced back to the corresponding items(s) in the EvDBT. Therefore, the version information of each atomic item in the EvDBT will be inherited by the corresponding item in the new diagram. Furthermore, since some of the projections are a many-to-one mapping, the version tag of the target item will be a joining of the version tags of each of the original items mapped to the targeted item.

To further clarify the duplicated node problem discussed above; this problem is caused by node movement. Once other evolutionary design diagrams are projected out from an EvDBT, said duplicated nodes will be merged back into one node that has the right version tag. Referring to the example in Fig. 12, the two $D$ nodes appeared in the EvDBT will become one in the EvCIN. This node will have the version tag of $\left\{v_{1}, v_{2}\right\}$. Therefore the node movement problem is handled gracefully in this approach.

\section{3 Formal Definition of the Tree Merging Process}

In the previous subsection, we have introduced the tree merging process through a simple example. In this section, we will apply graph theory to give a formal definition of the tree merging process and also prove that this process can be realized by scalable algorithms.

A graph is defined as a tuple $G=(V, E, s r c, \operatorname{tg} t)$, where $V$ is a set of nodes, $E$ is a set of edges and src, tgt : $E \rightarrow V$ are functions respectively giving the source node and target node of each edge.

A tree is a special type of graph. There are a number of equivalent definitions of trees [36]. A general tree may not need to specify the root node, but in this section, we only consider rooted unordered trees. A tree in this section is defined as follows: 
A tree is defined as a triple $T=\left(V, v_{0}\right.$, par $)$, where $V$ is a set of nodes, $v_{0}$ is the root node, par: $V \rightarrow V \cup\{\phi\}$ is a function giving the parent node of each node (here we ignore the trivial case of an empty tree). $\phi$ is an empty node. Based on the properties of a tree, we have:

$$
\operatorname{par}\left(v_{0}\right)=\phi ; \forall v \in V, \exists k<|V|, \operatorname{par}^{[k]}(v)=v_{0}
$$

In a tree, each edge is associated with a unique child node and can be determined by the function par, so the set of edges is removed from the definition of a tree to simplify the discussion.

Let $T=\left(V, v_{0}\right.$, par $)$ be a tree, without confusion, when we say a node $v \in T$, we mean $v \in V$. We also define $|T|=|V|$, that is the total number of nodes in the tree. For a node $v \in T$, we define the level of the node as $\operatorname{lev}(v)=k$ where $\operatorname{par}^{[k-1]}=v_{0}$ (according to this definition, the level of a root node is 1 ). The level of the tree is the maximum level of the node in the tree. The definition can be described as:

$$
\operatorname{lev}(T)=\min _{k}(k \in \mathbb{N}, \forall v \in T, \operatorname{lev}(v) \leq k)
$$

A labeled graph is a graph with label information for the nodes and edges. Similarly, a labeled tree is a tree with label information on the nodes.

Let $L$ be a set of labels. A labeled tree is defined as a tuple $L T=\left(V, v_{0}\right.$, par, $L$,label $)$, where label : $V \rightarrow L$ is a node labeling function.

In a behavior tree, each node is associated with a component and some behavior. We can use the name of the component and the corresponding behavior to define a naming function for the node. For example, if the node represents a component called "LIGHT" in a state called "[on]" it can be named as "LIGHT[on]". It is easy to define a naming function so different components or a same component with different behaviors will have different names.

Definition 3.1 Let $T$ be a behavior tree, name a naming function for every node in $T$, con a string concatenation function. Then we define the labeling function lab for $T$ as:

$$
\left\{\begin{array}{c}
\operatorname{lab}\left(v_{0}\right)=\operatorname{name}\left(v_{0}\right) \\
\operatorname{lab}(v)=\operatorname{con}(\operatorname{name}(v), \operatorname{lab}(\operatorname{par}(v))) \quad v \neq v_{0}
\end{array}\right.
$$

In a behavior tree, each parent node should not have two child nodes of the same behavior. To formalize this point, we have the following definition.

A behavior tree $T$ is called concise, while $\forall v_{a}, v_{b} \in T, v_{a} \neq v_{b}$, if $\operatorname{par}\left(v_{a}\right)=\operatorname{par}\left(v_{b}\right)$ then $\operatorname{name}\left(v_{a}\right) \neq \operatorname{name}\left(v_{b}\right)$.

Proposition 3.1: $T$ is a finite concise behavior tree, $l a b$ is the labeling function as in Definition 3.1, $\forall v_{a}, v_{b} \in T, v_{a} \neq v_{b}$, then $\operatorname{lab}\left(v_{a}\right) \neq \operatorname{la} b\left(v_{b}\right)$

Proposition 3.1 means for a concise behavior tree (we assume that a typical behavior tree should be concise), each node will have a unique label based on the labeling function defined in Definition 3.1. This proposition can be easily proved by using mathematical induction.

Now we need to add the version information into a behavior tree. Each node in an evolutionary behavior tree should carry some version information. The version information is similar to a type defined in a typed graph [45]. However, the difference between a typed graph and a versioned behavior tree is that a node in a versioned behavior tree is associated with a set of versions.

A versioned behavior tree $T$ is defined as a behavior tree plus a set of versions $V R$ and a version function ver where $\forall v \in T$, ver $(v) \subseteq V R$.

For example, if we have a behavior tree $T$, and we define the version of the tree as $v_{1}$, then the version set of the tree is $V R=\left\{v_{1}\right\}$, and the version function $v e r, \forall v \in T, \operatorname{ver}(v)=\left\{v_{1}\right\}$. 
After a versioned behavior tree has been defined, a new function called function set merge ( $f s m$ ) needs to be introduced before formally defining the tree merge function. This is similar to the set merge function discussed by Sabetzadeh and Easterbrook [59]. Even though the set merge function discussed by Sabetzadeh and Easterbrook's paper is slightly different from $f s m$, the intuitive description in that paper may be helpful in understanding the function set merge function ( $f_{s m}$.)

Definition 3.2: Let $f_{1}, \ldots, f_{k}$ are bijections from finite sets $S_{1}, \ldots S_{k}$ to finite sets $R_{1}, \ldots R_{k}$. R is the union of $R_{1}, \ldots R_{k}$ and $\Xi$ is the disjoint union $^{1}$ of $S_{1}, \ldots S_{k}, \Xi=\coprod_{i=1}^{k} S_{i}$. Then we can define a finite set $S$, a bijection $f$ and a surjective ${ }^{2}$ function $f s m$ that satisfy the following properties:

1. fsm: $\Xi \rightarrow S ; f: S \rightarrow R$

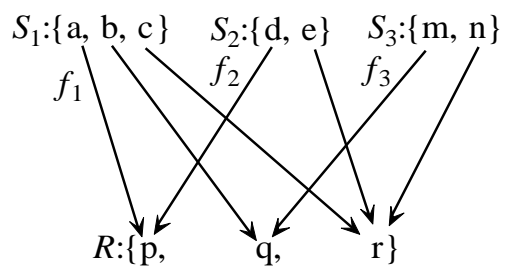

Fig. 13. Set $S_{1}, S_{2}, S_{3}$ and function $f_{1}, f_{2}$ and $f_{3}$.

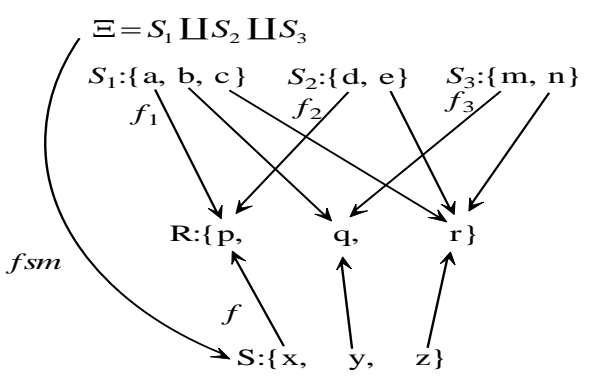

Fig. 14. Example of a function set merge.

2. $\forall x_{i} \in \Xi, x_{i} \in S_{i}$, then $f\left(f \operatorname{sm}\left(x_{i}\right)\right)=f_{i}\left(x_{i}\right)$.

3. $\forall x_{i}, \quad y_{j} \in \Xi, x_{i} \in S_{i}, y_{j} \in S_{j}, f \operatorname{sm}\left(x_{i}\right)=f \operatorname{sm}\left(y_{j}\right)$ if and only $f_{i}\left(x_{i}\right)=f_{j}\left(y_{j}\right)$;

We also denote $(S, f)=F S M\left(S_{1}, \ldots S_{k}, f_{1}, \ldots, f_{k}\right)$.

The following example helps readers to understand the definition of $f_{s m}$. As shown in Fig. 13, $S_{1}=\{\mathrm{a}, \mathrm{b}, \mathrm{c}\}, S_{2}=\{\mathrm{d}, \mathrm{e}\}, S_{3}=\{\mathrm{m}, \mathrm{n}\}$ are three sets; $f_{1}, f_{2}$ and $f_{3}$ are three bijections on the three sets with $R=\{\mathrm{p}, \mathrm{q}, \mathrm{r}\}$ as the union of the codomains. The mapping of the three functions is listed in Table 1 .

Then the merged set $S=\{\mathrm{x}, \mathrm{y}, \mathrm{z}\}$ and the bijection $f$ are shown in Fig. 14. The mapping of $f s m$ and $f$ is shown in Table 2. Please note that the $f s m$ is a many to one mapping, so the result of $f s m^{-1}$ is a set. The example above shows:

$$
f s m^{-1}(\mathrm{x})=\{\mathrm{a}, \mathrm{d}\} ; f s m^{-1}(\mathrm{y})=\{\mathrm{b}, \mathrm{m}\} ; f s m^{-1}(\mathrm{z})=\{\mathrm{c}, \mathrm{e}, \mathrm{n}\} .
$$

After the function set merge is defined, we can give a formal definition of the versioned behavior tree merge. A limitation of the tree merge is that the root node for each merged tree must be identical.

Definition 3.3: Let $T_{1}, T_{2}, \ldots T_{k}$ be concise versioned behavior trees, $T_{i}=\left(V_{i}, v_{0}\right.$, par $_{i}, L_{i}$, lab $\left._{i}, V R_{i}, v e r_{i}\right), i=1, \ldots, k$. The versioned tree merge function $M$ is defined as: $M\left(T_{1}, \ldots T_{k}\right)=T$,

TABLE 1 THE MAPPING OF FUNCTION $f_{1}, f_{2}, f_{3}$

$$
T=\left(V, v_{0}, \text { par, } L, l a b, V R, v e r\right)
$$

where:

1. $L=L_{1} \cup L_{2} \cup \cdots \cup L_{k}$

2. $V R=V R_{1} \cup V R_{2} \cup \cdots \cup V R_{k}$

3. $(V, l a b)=F S M\left(V_{1}, \ldots, V_{k}, l a b_{1}, \ldots, l a b_{k}\right)$

\begin{tabular}{|c|c|c|c|c|c|c|c|}
\hline & $\mathrm{a}$ & $\mathrm{b}$ & $\mathrm{c}$ & $\mathrm{d}$ & $\mathrm{e}$ & $\mathrm{m}$ & $\mathrm{n}$ \\
\hline$f_{1}$ & $\mathrm{p}$ & $\mathrm{q}$ & $\mathrm{r}$ & - & - & - & - \\
\hline$f_{2}$ & - & - & - & $\mathrm{p}$ & $\mathrm{r}$ & - & - \\
\hline$f_{3}$ & - & - & - & - & - & $\mathrm{q}$ & $\mathrm{r}$ \\
\hline
\end{tabular}

4. $\operatorname{par}\left(v_{0}\right)=\phi ; \forall v \in V, v \neq v_{0}$, let $v_{i} \in f \mathrm{sm}^{-1}(v), v_{i} \in V_{i}$, then $\operatorname{par}(v)=f \operatorname{sm}\left(\operatorname{par}_{i}\left(v_{i}\right)\right)$.

5. $\forall v \in V, \operatorname{ver}(v)=\bigcup_{v_{i} \in f s m^{-1}(v)} \operatorname{ver}_{i}\left(v_{i}\right)$.

\begin{tabular}{|c|c|c|c|c|c|c|c|}
\hline & $\mathrm{a}$ & b & c & d & e & $\mathrm{m}$ & $\mathrm{n}$ \\
\hline$f_{s m}$ & $\mathrm{X}$ & $\mathrm{y}$ & Z & $\mathrm{X}$ & Z & $\mathrm{y}$ & Z \\
\hline & & & & & & Z & \\
\hline$f$ & & & & & & $r$ & \\
\hline
\end{tabular}

TABLE 2 THE MAPPING OF FUNCTION $f s m$ AND $f$

\footnotetext{
1 a disjoint union is a modified union operation which indexes the elements according to their origin sets.

2 A function $f: X \rightarrow Y$ is surjective if and only if for every $y$ in the codomain $Y$ there is at least one $x$ in the domain $X$ such that $f(x)=y$.
} 
Definition 3.3 defines the merge process for versioned behavior trees. The merge process includes five steps. step 1 and 2 are set unions; step 3 is a function set merge. Step 4 indicates that the function of par is preserved by the function of $f s m$ and the last step is a composition of set union and $f s m$. Next, we will prove that the tree merging function is commutative. In order to prove this, we need to prove two propositions first.

Proposition 3.2: Set union is commutative.

Proof: Let $S_{1}, S_{2}$ be two sets. $S=S_{1} \cup S_{2}$ and $S^{\prime}=S_{2} \cup S_{1} . \forall s \in S$, we have $\left(s \in S_{1}\right) \vee\left(s \in S_{2}\right)$, so we know $s \in S^{\prime}$, and $S \subseteq S^{\prime}$. Similarly, $S^{\prime} \subseteq S$. Therefore $S=S^{\prime} . \square$

In the similar way, we can prove that disjoint union is also commutative.

Proposition 3.3: Function set merge is commutative.

Proof: Let $S_{1}, S_{2}$ be two finite sets, $f_{1}, f_{2}$ be two bijections, $f_{i}: S_{i} \rightarrow R_{i}, i \in\{1,2\}$, $(S, f)=\operatorname{FSM}\left(S_{1}, S_{2}, f_{1}, f_{2}\right),\left(S^{\prime}, f^{\prime}\right)=\operatorname{FSM}\left(S_{2}, S_{1}, f_{2}, f_{1}\right)$

1. Because both set union and disjoint union are commutative, $\Xi=S_{1} \amalg S_{2}=S_{2} \amalg S_{1}=\Xi^{\prime}$, $R=R_{1} \cup R_{2}=R_{2} \cup R_{1}=R^{\prime}$.

2. According to Definition 3.2, fsm, $S$ and $f$ depend only on the mapping tables of $f_{1}$ and $f_{2}$, but not on the order of $f_{1}$ and $f_{2}$, so $S=S^{\prime}, f=f^{\prime}$.

Therefore Function set merge is commutative.

Theorem 3.1: The tree merging function defined in Definition 3.3 is commutative.

Proof: Let $T_{1}=\left(V_{1}, v_{0}\right.$, par $\left._{1}, L_{1}, l a b_{1}, V R_{1}, v e r_{1}\right)$ and $T_{2}=\left(V_{2}, v_{0}\right.$, par $\left._{2}, L_{2}, l a b_{2}, V R_{2}, v e r_{2}\right)$ be two concise versioned trees. $T=M\left(T_{1}, T_{2}\right)$ and $T^{\prime}=M\left(T_{2}, T_{1}\right)$. We need to prove $T=T^{\prime}$.

Let $T=\left(V, v_{0}\right.$, par, $\left.L, l a b, V R, v e r\right) \quad, T^{\prime}=\left(V^{\prime}, v_{0}\right.$, par' $\left.^{\prime}, L^{\prime}, l a b^{\prime}, V R^{\prime}, v e r^{\prime}\right)$.

Because the tree merge does not change the root node of the merged trees, we keep $v_{0}$ as the root node for $T$ and $T^{\prime}$.

1. According to Definition 3.3, $L=L_{1} \cup L_{2}, L^{\prime}=L_{2} \cup L_{1}$. Based on Proposition 3.2, we know that $L=L^{\prime}$; similarly, $V R=V R^{\prime}$.

2. $(V, l a b)=\operatorname{FSM}\left(V_{1}, V_{2}, l a b_{1}, l a b_{2}\right) \quad$ and $\quad\left(V^{\prime}, l a b^{\prime}\right)=F S M\left(V_{2}, V_{1}, l a b_{2}, l a b_{1}\right) \quad$. From Proposition 3.3, we know that $V=V^{\prime}$ and $l a b=l a b^{\prime}$.

3. $\forall v \in V$ (we already know $V=V^{\prime}$ ), if $v=v_{0}, \operatorname{par}\left(v_{0}\right)=\operatorname{par}^{\prime}\left(v_{0}\right)=\phi$. If $v \neq v_{0}$, because $(V, l a b)=F S M\left(V_{1}, V_{2}, l a b_{1}, l a b_{2}\right), \exists v_{a} \in V_{i}, \quad i \in\{1,2\}, \quad v=f \operatorname{sm}\left(v_{a}\right)$. Based on the definition, $\operatorname{par}(v)=f \operatorname{sm}\left(\operatorname{par}_{i}\left(v_{a}\right)\right)$ and also $\operatorname{par}^{\prime}(v)=f \operatorname{sm}\left(\operatorname{par}_{i}\left(v_{a}\right)\right)$. Because both $\operatorname{par}_{i}$ and $f s m$ are many-to-one mapping, $\operatorname{par}(v)=\operatorname{par}^{\prime}(v)$. Then we know $\operatorname{par}=\operatorname{par}^{\prime}$.

4. Based on the definition, $\forall v \in V, \operatorname{ver}(v)=\bigcup_{v_{i} \in f s m^{-1}(v)} v e r_{i}\left(v_{i}\right), i \in\{1,2\}$. Because set union is commutative (Proposition 3.2). We have $\operatorname{ver}(v)=\operatorname{ver}^{\prime}(v)$.

Combining the results above, we have $T=T^{\prime} . \square$

Next we will prove that when the number of merged trees is smaller than a constant number $k$ and $n$ is the size of the largest merged tree, then the tree merge function can be computed in the time of $O(n \log n)$.

Theorem 3.2: Let $T_{1}, T_{2}, \ldots T_{k}$ be concise versioned behavior trees that satisfy the following conditions:

1. $\forall 1 \leq i \leq k,\left|T_{i}\right| \leq n$

2. $k \leq c, c$ is a constant; 
Let $T=M\left(T_{1}, \ldots T_{k}\right)=\left(V, v_{0}\right.$, par, L, lab, VR, ver $)$

Then there exist an algorithm to compute the version merged tree $T$ in time of $O(n \log n)$.

Proof: Let us consider the five steps of the version tree merging algorithm:

1. $L=L_{1} \cup L_{2} \cup \cdots \cup L_{k},|L| \leq \sum_{1 \leq i \leq k}\left|L_{i}\right| \leq k \times n$. There exist sorting algorithms to sort the elements in all the input sets and merge the same elements to generate the target set in the time of $O(k \times n \times \log (k \times n))$ [36]. Because $k \leq c(c$ is a constant), the sorting algorithm can finish the set merge in $O(n \log n)$.

2. $V R=V R_{1} \cup V R_{2} \cup \cdots \cup V R_{k}$. Proof as step 1 .

3. $(V, l a b)=F S M\left(V_{1}, \ldots, V_{k}, l a b_{1}, \ldots, l a b_{k}\right)$. For this operation, the main point is that for each node in one of the input trees, we need to identify all the nodes in other trees with the same label. In each input tree, we can sort all the nodes based on their labels. Obviously, we can have a sorting algorithm finish the sorting in $O(n \log n)$. After all the nodes are sorted according to the label, to search a node with a given label in an input tree may take $O(\log n)$ time. Finally, going through all the nodes in the input trees will take $O(n \log n)$ in time.

4. $\operatorname{par}\left(v_{0}\right)=\phi, \forall v \in V, v \neq v_{0}, v_{a} \in f s m^{-1}(v)$. If $v_{a} \in V_{i}$, then $\operatorname{par}(v)=f s m\left(\operatorname{par}_{i}\left(v_{a}\right)\right)$. It is a composition of $f s m$ and par $_{i}$. Because par $_{i}$ is a finite mapping function, it can be finished in $O(1)$. Therefore, the total time is under $O(n \log n)$ in time.

5. $\forall v \in V, \operatorname{ver}(v)=\bigcup_{v_{i} \in f s m^{-1}(v)} v e r_{i}\left(v_{i}\right)$. Because $\forall v \in V, \operatorname{ver}(v) \subseteq V R,|\operatorname{ver}(v)| \leq|V R|=k \leq c$, the operation to compute $v e r(v)$ is $O(1)$ and the total time is under $O(n \log n)$

Because each of the five steps can be computed in $O(n \log n)$ in time, there exist algorithms to merge the trees in $O(n \log n)$ in time. Compared to a state-chart merging algorithm with time complexity of $O\left(n^{2}\right)$ [50], there exists more efficient tree merging algorithms.

Theorem 3.2 has proven that there is a way to compute the versioned tree merging algorithm in $O(n \log n)$ time. This result is important because it shows that the tree merge algorithm is at least theoretically scalable.

In the case of large systems, it is possible that the number of all versions is correspondingly large. When a reviewer seeks to understand how the system has evolved, he may simply select a representative sample of typical versions to compare. Which versions to select for comparison will depend on the purpose of the exercise. For example, if the reviewer wants to see the overall evolution of the system, he may select the versions of some major releases. If he wants a highergranularity view of a particular part of the system, he may select only versions in which the interested part had been updated. (Of course, the task to identify a small set of relevant versions could be difficult if the system has a large number of different versions. However, with suitable software tools that can scan and identify the changed section of a version, this task could be significantly simplified.) Therefore, the condition of $k \leq c$ can be held for most practical cases.

\section{Case Studies}

In this section, two case studies are introduced to illustrate the concept and the scalability of the traceability model. The case studies are investigated by using a software environment called "Integrare" [78],

"Integrare" integrates a number of different software tools to support the BE approach. It implements the traceability model. This feature has been demonstrated elsewhere [79]. All of the diagrams shown in this section are screenshots captured while using "Integrare". 


\subsection{Microwave Oven System}

The case study concerns the microwave oven system, previously introduced in the second section. The original microwave oven case study included the seven stated functional requirements. The requirements and the design have been introduced in the second section. The original design is the first version $\left(v_{1}\right)$.

In this subsection, we will change the requirements twice and create two new versions of design. The two new versions are labeled as $v_{2}$ and $v_{3}$ respectively.

In the first change of the software requirements, two transitory behaviors of OVEN, OVEN:[Cooking-Stop] and OVEN:[Cooking-Finished] have been removed. Then a new requirement $\mathrm{R} 8$ is added.

R8: When the oven is idle, if the user opens the door, the door is open; the oven will be in the state of open.

Then the requirements are changed for a second time. This time, a new component TIMER is added to fulfill the timing functions, which were originally provided by the OVEN component. This change requires the original requirements 1,2 and 6 to be rewritten as below (the modified parts are underlined). It represents a design refinement as it involves the introduction of a new component, TIMER, not mentioned in the original requirements that were used to construct the formal specification.

Modified R1: There is a single control button available for the user of the oven. If the oven is idle with the door closed and you push the button, the timer will be set to one minute, and the oven will start cooking (that is, energize the power-tube).

Modified R2: If the button is pushed while the oven is cooking it will cause the timer to add one extra minute.

Modified R7: If the timer times-out, the light and power-tube are turned off and then a beeper emits a sound to indicate that the cooking is finished.

The second and the third versions of the DBT are shown in Fig. 15 and Fig. 16.

The next step is to merge the three DBTs and create the EvDBT shown in Fig 17. In this figure, the new fragments of behavior (behaviors that only exist in $v_{3}$ ) are drawn with bold lines, the old fragments of behavior, which are not in $v_{3}$, are drawn in gray and the unchanged parts (behaviors in both $v_{2}$ and $v_{3}$ ) are drawn in the normal style. In Integrare, when the mouse moves over a node or a link, the version information is displayed in the tool tip.

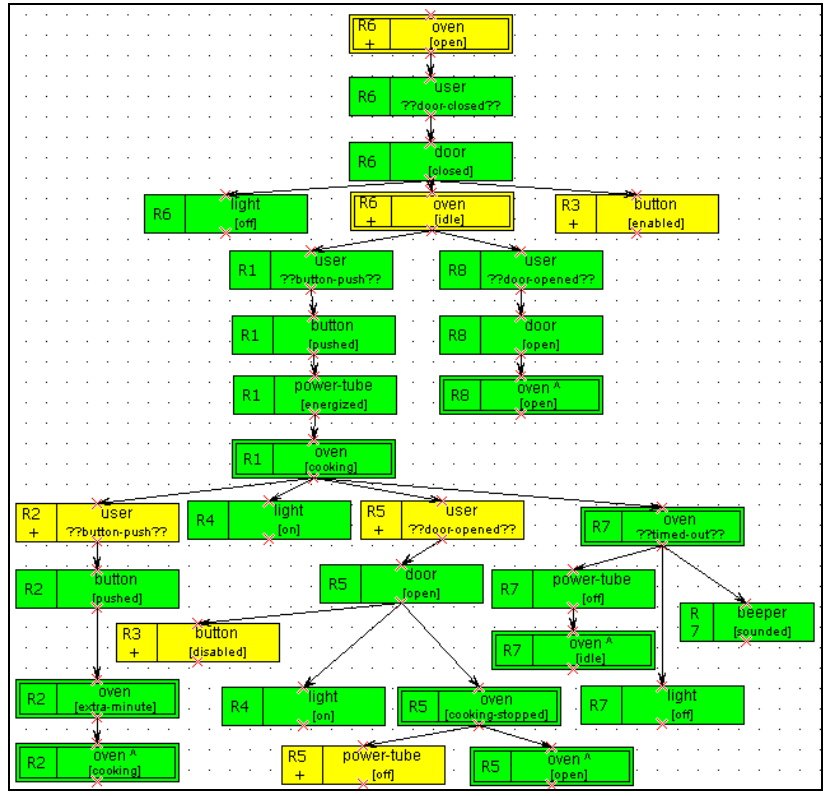

Fig. 15. The DBT of the second version (v2).

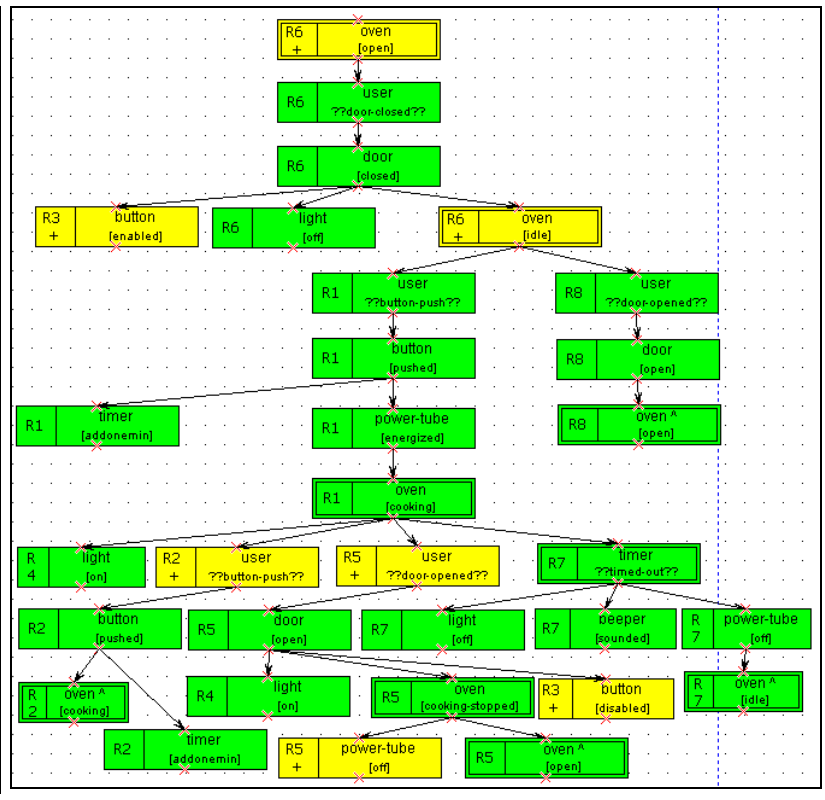

Fig. 16. The DBT of the third version $\left(v_{3}\right)$. 


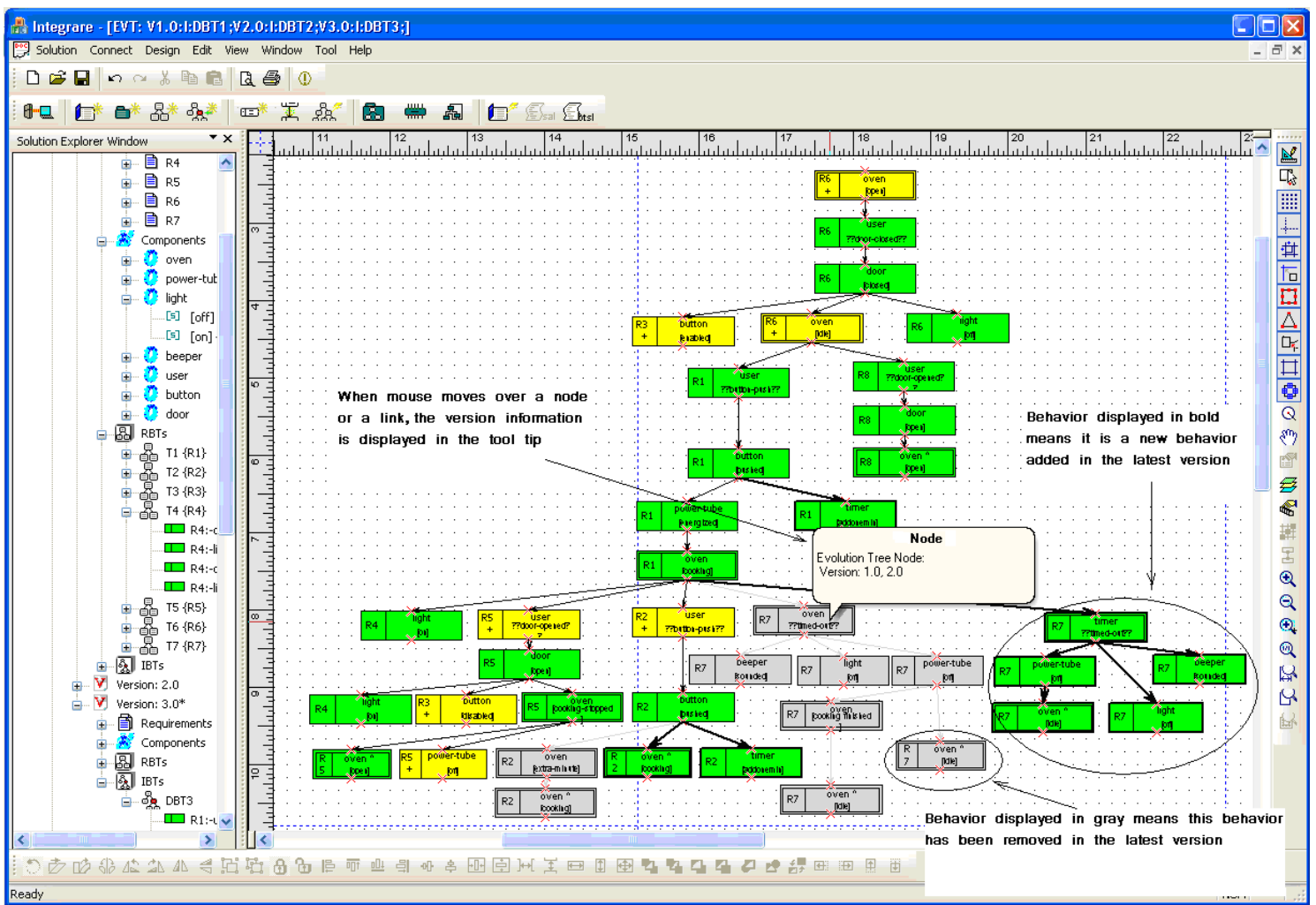

Fig. 17. The EvDBT of the microwave oven system

From the EvDBT, other evolutionary diagrams are projected as shown in Fig. 18. Because of space limitations, only the EvCIN and the EvCBT of the component OVEN are presented.

Fig. 18 (left part) shows the EvCIN; the change impact and the evolutionary information for the architecture are marked by associating each component and each dependency relationship with a set of version tags. From it, we can see that a new component TIMER is added in $v_{3}$ while the other components exist in all the three versions. Similarly, several interaction relationships between the component OVEN and other components are removed, and several component interaction relationships with TIMER are introduced in $v_{3}$. Introduction of the TIMER component represents a design decision.

Fig. 18 (right part) is the EvCBT of component OVEN. As with our other evolutionary diagrams, each atomic item in this diagram has attached a set of version tags. From these version tags, the evolutionary status of a component's internal behavior is recorded and can be readily traced.

This example demonstrates how the proposed model can be used to identify change impacts. It records evolutionary history and traces the requirements changes and the corresponding changes to

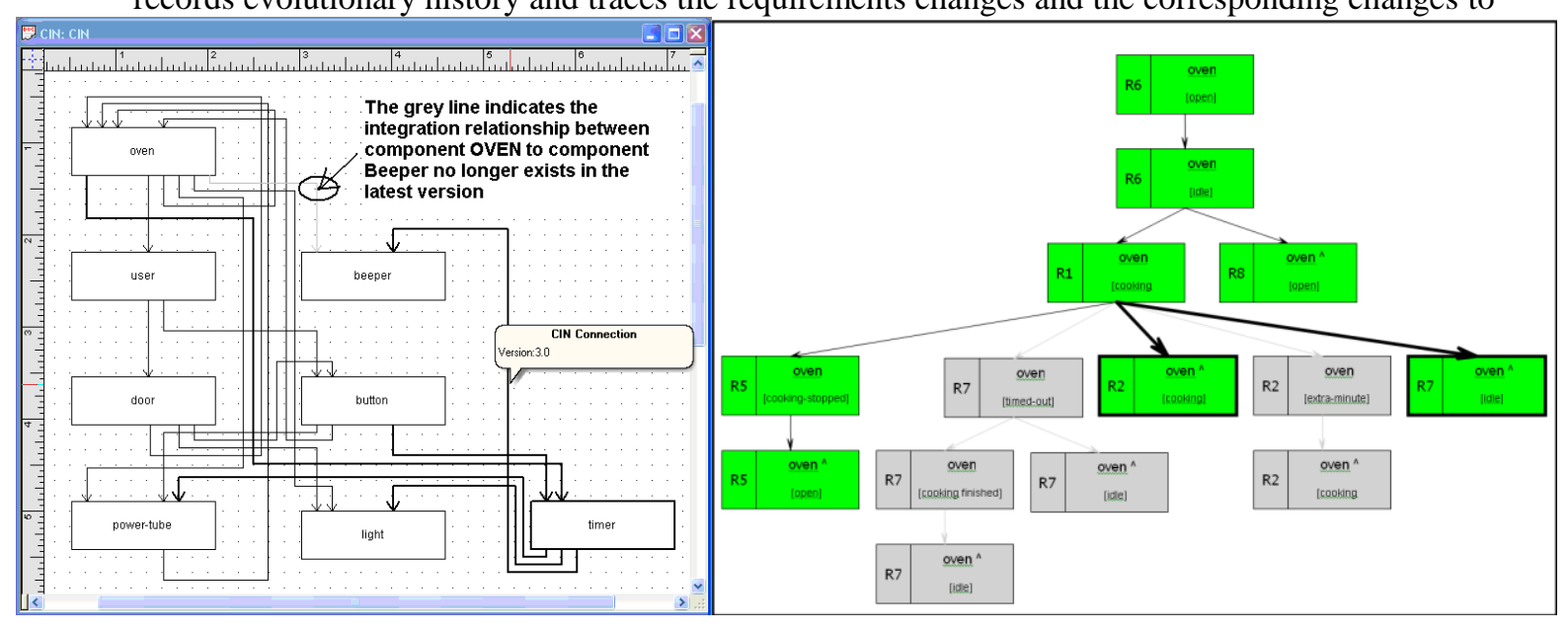

Fig. 18. The EvCIN of the microwave oven system (left) and the The EvCBT of component OVEN designs for different artifacts in a software system at the architecture level and the component 
level. Each evolutionary diagram shows the information of all three different versions. In a real software system, the number of versions is likely to be many. In such cases the software tools can help users to select the versions of interest and display the information in a configurable way.

\section{2 The COMBIZ Satellite Control System}

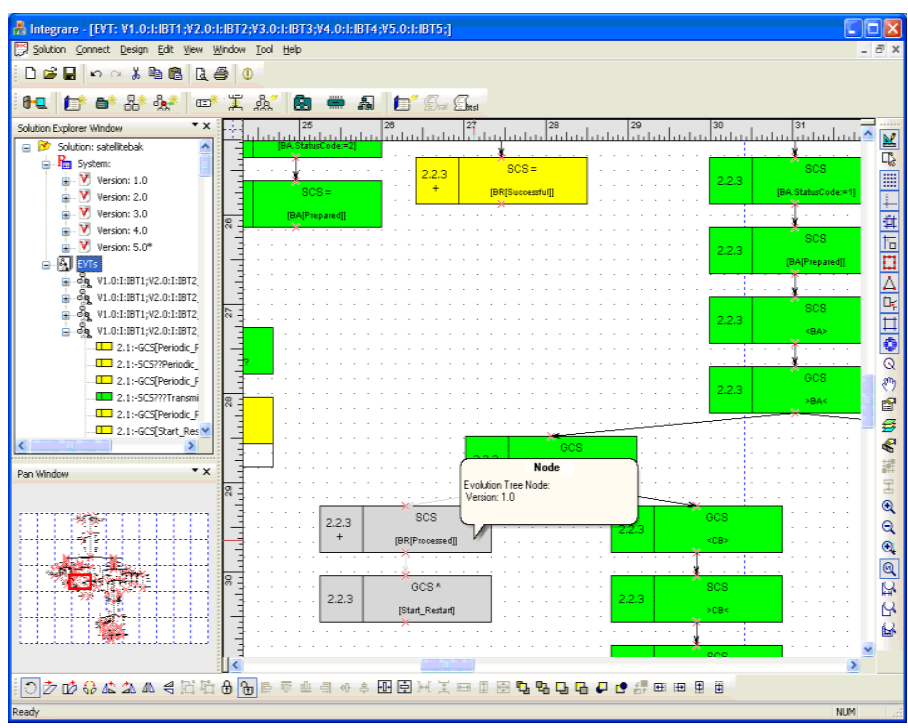

Fig. 19. Part of the EvDBT of the Satellite Control System

The COMBIZ Satellite Control System case study gives a detailed example of the BE methodology in action and is indicative of its capabilities.

The purpose of the Satellite Control System is to manage the system's satellite assets to guarantee the reliable throughput of communication packets from uplink to downlink satellite data communication sites within that customer's allotted time slot and to minimize the amount of time required for allocation to maintenance time slots. The operational demands for this system are exacting, with high-reliability being of paramount importance.

The data communication assets are managed by a Ground Control System (GCS). The GCS is responsible for the health and maintenance of the Satellite Control System (SCS) as well as for scheduling bulk data communication time slots for its customers. The SCS is the primary interface for COMBIZ's satellite bulk data transmission customers who operate satellite data communication uplink and downlink sites.

The COMBIZ's satellite constellation consists of three satellites, each with an 8-hour contact window with one of three GCS ground sites. Thus, there is 24 -hour satellite coverage to address all ground control system operations and customer data communication requirements. The three satellites and their hardware and software systems represent the SCS. The three ground control sites represent the Ground Control System.

The GCS directs the SCS activities on the basis of pre-scheduled time slots. Time slots are allocated for maintenance activities and customer data transmission preparation, e.g., pointing a satellite by executing a firing command or updating the SCS's broadcast/location table. Time slots are also allocated for customer data transmission. After system initialization, a maintenance time slot is always performed, followed by a customer data transmission time slot, followed by another maintenance time slot. Maintenance time slots and customer data transmission time slots are performed in alternating order. We refer to the activities performed during a maintenance time slot as a "Manage Satellites" transaction. We refer to the activities performed during a customer data transmission time slot as a "Transmit Data" transaction.

The original requirements of the COMBIZ Satellite Control System are derived from three sources [38]. The complete document, which includes the background, introduction, terminology and requirements, is 14 pages [65], too long to include in this paper, but nonetheless important background material. This full case study is shown in the website [66].

The COMBIZ Satellite Control System consists of a Ground Control System (GCS). It includes three ground sites and three satellites. The three satellites and their hardware and software represent the SCS.

The system provides its internal and external customers with a satellite-enabled data communications service. The GCS directs the SCS activities for maintenance activities and customer data transmission preparation, e.g., pointing a satellite by executing a firing command or updating the SCS's broadcast/location table. 
As can be seen in the on-line case study, the original document includes 10 major requirements, which are composed of about 60 detailed requirements. We have changed the requirements 4 times and created 5 different versions of the DBT and then merged them together by using "Integrare". Using this software tool, it was possible to merge the five DBT's and generate a new tree in less than one second.

The final EvDBT contains about 180 nodes, requiring around 20 A4 pages to print out in the normal size. Due to this quantity of information, it is impractical to provide a detailed readable version of the tree in this paper. However, all details of the requirement changes, DBTs of different versions and a detailed readable image of EvDBT can be found at the website [66]. Fig. 19 is part of the EvDBT. From this figure, we find out that by applying a suitable tool such as "Integrare", designers can retrieve and trace all the evolution information easily.

Fig 20 shows a small part of the EvDBT. From this figure, the maintainer can easily obtain the fact that one of the difference between version 5 and other versions is that the SCS needs to "Remove Entries" before sending out "TC" when it "Transmit_Data". It serves as a simple example to show how this approach may help to understand change.

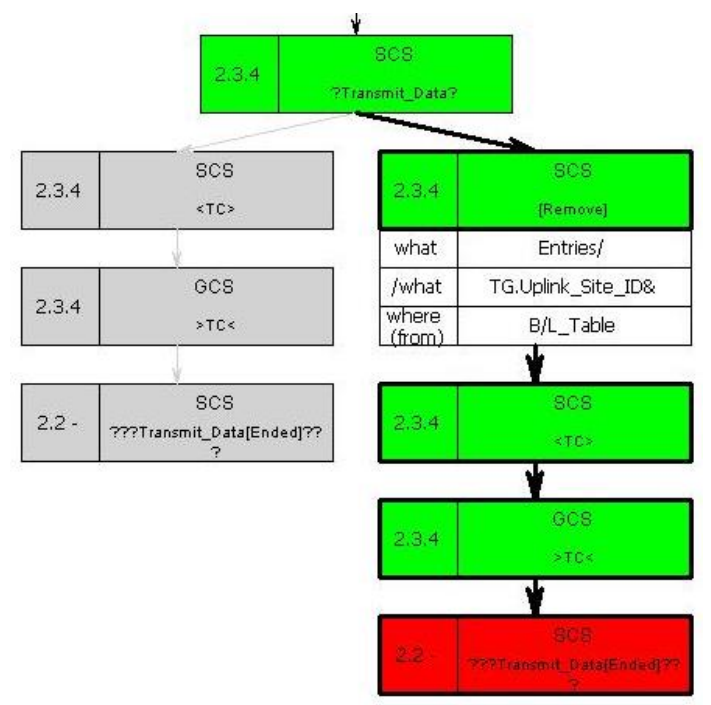

Fig. 20. Example of how the EvDBT help to understand change

\section{3 Indicative Performance Measurement}

We have used both the microwave oven case study and the satellite control system to measure the performance of the tree merging algorithm implemented by Integrare (running on a Dell D800 laptop computer). The indicative results are shown from Table 3 to Table 6 . These results are encouraging and point towards the need for more detailed performance measurement in future studies.

Table 3 shows that the numbers of nodes of the microwave over system's DBTs are 28 (the first version), 30 (the second version), and 31 (the third version). The size and time used to generate the EvDBTs are listed in Table 4. To merge the DBT of the first version and the second version results an EvDBT of 32 nodes; the time used to generate the EvDBT is 50 milliseconds. The test results of the satellite control system are listed in Table 5 and Table 6. From these results, we have found: 
1. Increasing the size of the merged DBTs will increase the time used to merge them. They are close to a linear relationship.

2. Table 6 shows that the time used to merge the trees increases when the number of merged trees is increased. However, the number of merged trees has less impact on the merging time compared to the size of the trees.

The testing results are consistent with the theoretical prediction.

\section{Conclusion}

\section{and Future Work}

This paper introduced a high performance traceability model, an extension of our previous work [77]. The model offers a new approach to software change impact analysis [6] at the architecture and the component levels. It differs from other dependency analysis [70] and change impact analysis methods [85], in the way the traceability model comprehensively maps changes from the problem domain to the solution domain, a feature not available in other approaches. Moreover, the extended model can handle multiple sets of changes at different times and be able to generate different types evolutionary design documents. This means it can provide traceability in the horizontal direction as well as in the vertical direction (Fig. 1).

It remains to be seen that maintaining a software system using this extended traceability method might have the potential to increase the length of the Evolution stage [5] of a system, and therefore increase the lifespan of the system, thus deriving the financial benefits of an extended life-span. An advantage of the model is that all the model transformations are based on formally described rewrite rules and can be supported by software tools [78]. As some non-functional requirements such as safety and security requirements can be modeled by behavior trees [83], this model may also contribute to the management of some non-functional requirements. It might also help to optimize software architecture, because the designer could quickly identify the change impact of a new possible design for the system. The designer may try different possible change solutions before finalize a most suitable change solution.

Since the traceability model introduced in this paper is based on the restriction of using unique identifiers for components and behaviors, a possible extension of this work is to explore the development of an advanced feature that supports even when some constituent components or behaviors are renamed [1].

Another possible research direction is to study automatic transformations from the Behavior Tree models into implementation languages. While the traceability model presented in this paper is designed specifically for the BE approach, the improvement of the tree merging algorithm by applying general graph merging algorithms [59] or graph rewrite grammars such as triple graph grammars (TGG) [27], the traceability model might be extended to systems designed using other approaches. 


\section{Acknowledgment}

The authors would like to acknowledge the Australian Research Council (ARC) Centre for Complex Systems for its support of this work. The authors would also like to thank Associate Professor Alan Liew for his review of this paper.

\section{Special Notice}

During the preparation of this paper, the third named author R. Geoff Dromey passed away.

\section{References}

[1] Abi-Antoun, M., Aldrich, J., et al., "Differencing and Merging of Architectural Views", Proceedings of the 21st IEEE/ACM International Conference on Automated Software Engineering, pp 47-58, 2006

[2] Alanen, M., Porres, I., "Difference and Union of Models", TUCS Technical Report No 527, Turku Centre for Computer Science, 2003

[3] Bass, L., Clements, P. and Kazman, R., "Software Architecture in Practice", ISBN: 0201199300, Addision Wesley Longman, Inc. 1998

[4] Behavior engineering web site, Behavior Engineering, http://www.beworld.org

[5] Bennett, K., Rajlich, V., "Software Maintenance and Evolution: A Roadmap", The Future of Software Engineering, Anthony Finkelstein (Ed.), ACM Press 2000

[6] Bohner, S. A., Arnold, R. S., "Software Change Impact Analysis", IEEE Computer society Press Los Alamitos, California, ISBN 0818673842, 1996

[7] Bouquet, F., Jaffuel, E., Legeard, B., Peureux, F., and Utting, M., "Requirements Traceability in Automated Test Generation Application to Smart Card Software Validation", Proceedings of the $1^{\text {st }}$ international workshop on advances in model-based testing, pp.1-7, 2005

[8] Buckley, J., Mens, T., Zenger, M., Rashid, A., and Kniesel, G., "Towards a Taxonomy of Software Change", Journal of Software Maintenance and Evolution: Research and Practice, v(17), pp. 309-332, 2005

[9] Bratthall, L., Johansson, E., Regnell, B., "Is a Design Rationale Vital when Predicting Change Impact? - A Controlled Experiment on Software Architecture Evolution”, PROFES 2000 - Second International Conference on Product Focused Software Process Improvement, Oulo, Finland, pp.126139,2000

[10] [Chapin, N., Hale, J. E., Khan, K. M., Ramil, Tan, W., "Types of Software Evolution and Software Maintenance", Journal of Software Maintenance and Evolution: Research and Practice, pp. 3-30, $\mathrm{v}(13), 2001$

[11] [Chung, L., Nixon, B. and Yu, E., "Using Non-Functional Requirements to Systematically Select Among Alternatives in Architectural Design", Proceedings of 1st International Workshop on Architectures for Software Systems, Seattle, pp. 31-43, 1995.

[12] [Colvin, R., Hayes, I.J., "A Semantics for Behavior Trees", ACCS Technical Report, No. ACCS-TR07-01, ARC Centre for Complex Systems, 2007.

[13] Conklin, J., "Hypertext: An Introduction and Survey", Computer, Sept. pp. 17-41, 1987,

[14] Cooper, L., Chung, L., "Managing Change in OTS-Aware Requirements Engineering Approach", ICSE-MPEC'05, pp.1-4, 2005

[15] Dromey, R.G., "From Requirements to Design: Formalising the Key Steps", (Invited Keynote Address), IEEE International Conference on Software Engineering and Formal Methods, SEFM'2003, pp. 2-11, Brisbane, September, 2003

[16] Dromey, R.G, Powell, D., "Early Requirements Defects Detection", TickIT International, 4Q05, pp. 3-13, 2005

[17] Dromey, R.G., "Formalizing the Transition from Requirements to Design", in Mathematical Frameworks for Component Software, Models for Analysis and Synthesis, Chapter 6, World Scientific, Ed. Liu, Z., and He, J., ISBN 981-270-017-X, pp. 173-206, 2006

[18] Dromey, R.G., "Scaleable Formalization of Imperfect Knowledge", 1st Asian Working Conference on Verified Software (AWCVS'06), pp.21-33, Macau, 2006

[19] Dromey, R.G., "Genetic Design: Amplifying Our Ability to Deal With Requirements Complexity", in S.Leue, and T.J. Systra, Scenarios, Lecture Notes in Computer Science, LNCS 3466, pp. 95 - 108, 2005 
[20] Dromey, R.G., "Engineering Large-Scale Systems - Mitigating the Pivotal Risks", IEEE Software (submitted)

[21] Förtsch, S.; Westfechtel, B.: "Differencing and Merging of Software Diagrams - State of the Art and Challenges"; in: Proc. Second Intl. Conf. Software and Data Technologies (ICSOFT 2007), Volume SE, Barcelona, Spain, July 22-25, 2007; INSTICC Press; 2007

[22] Fowler, M., Scott, K., UML Distilled (2nd Edition), Addison-Wesley, Canada, 2000.

[23] Gallagher, K.D., Lyle, J.R., "Using Program Slicing in Software Maintenance”, IEEE Transactions on Software Engineering, V17(8), pp751-761, 1991

[24] Garg, P. K., Scacchi, W., “A Hypertext System to Manage Software Life-Cycle Documents”, IEEE Software, Vol. 7, No.3, pp. 90-98, 1990

[25] Gonzalez-Perez, C., Henderson-Sellers, B., Dromey, G., "A Metamodel for the Behavior Trees Modelling Technique”, Third International Conference on Information Technology and Applications, ICITA 05, pp. 35-39, 2005

[26] Grunske, L., Lindsay, P., Yatapanage, N., and Winter, K., “An Automated Failure Mode and Effect Analysis Based on High-Level Design Specification with Behavior Trees", the Fifth International Conference on Integrated Formal Methods (IFM'05), pp. 129-149, 2005

[27] Grunske, L., Geiger, L., Lawley, M., "A Graphical Specification of Model Transformations with Triple Graph Grammars", First European Conference Model Driven Architecture - Foundations and Applications, ECMDA-FA 2005, Nuremberg, Germany, November 7-10, 2005, Lecture Notes in Computer Science Volume 3748, pp. 284-298, 2005

[28] Grunske L., "Early Quality Prediction of Component-Based Systems - A Generic Framework", Journal of Systems and Software, Elsevier, Volume 80, Issue 5, pp. 678-686, 2007

[29] Grunske L., Winter K. Colvin: R. "Timed Behavior Trees and their Application to Verifying Realtime Systems", In Proc. of 18th Australian Conference on Software Engineering (ASWEC 2007), pp. 211-222, 2007

[30] Harel, D., Politi, M., "Modeling Reactive Systems with Statecharts”, McGraw-Hill, N.Y 1998.

[31] Harjani, D., R., Queille, J. P., "A process model for the Maintenance of Large Space Systems Software", Proceedings of Conference on Software Maintenance, IEEE Computer society Press, pp. 127-136, 1992

[32] Horowitz, E., Williamson, R. C., "SODOS: A Software Documentation Support Environment - Its Definition", IEEE Trans. Software Eng., Vol. SE-12, No. 8, pp. 849-859, 1986

[33] IEEE. "IEEE Standard for Software Maintenance (IEEE Std 1219-1998)", 1998

[34] ISO/IEC, "Software Engineering - Software Maintenance", International Standards Organization, 1999

[35] Kitchenham, B.A., Travassos, G.H., Mayrhauser, Av, Niessink, F., Schneidewind, N.F., Singer, J., Takada, S., Vehvilainen, R., Yang, H., "Toward an Ontology of Software Maintenance", Journal of Software Maintenance, 11(6):365-389, 1999

[36] Knuth, D. E., "The Art of Computer Programming, Fundamental Algorithms", 3rd edition, Vol 1, ISBN 0201896834, Addison Wesley Longman, 1997

[37] Kolovos, Dimitrios S.; Ruscio, Davide Di; Pierantonio, Alfonso; Paige, Richard F., "Different Models for Model Matching: An Analysis Of Approaches To Support Model Differencing" p.1-6 in: Proc. 2009 ICSE Workshop on Comparison and Versioning of Software Models, May 17, 2009, Vancouver; IEEE; 2009

[38] Krell, B.E., "Developing with Ada: Life Cycle Methods”, Bantam Books, New York, 1992.

[39] Lehman, M. M., "Program Evolution", Academic Press, London, pp.75-87,1985

[40] Lehman, M. M., Ramil, J., Wernick, P., Perry, D. E., Turski, W., M., "Metrics and Laws of Software Evolution - the nineties view", Proceedings 4th International Symposium on Software Metrics, pp. 20-32, 1997

[41] Lehner, F., "Software Lifecycle Management Based on a Phase distinction Method", Microprocessing and Microprogramming, vol. 32 pp. 603-608, 1991

[42] Lin, K., Chen, D. Sun C. and Dromey, R.G., "Maintaining constraints in collaborative graphic systems: the CoGSE approach", 9th European Conference on CSCW, Paris, France, pp.185-204, Sept. 2005

[43] Lindvall, M. and Sandahl, K., "Practical Implications of Traceability" Software Practice and Experience, vol. 26, no. 10, pp. 1161-1180, 1996.

[44] Luckham, D. C., Kenney, J.J., Ausgusin, L.M., Vera, J., Bryan, D., and Mann, W., "Specification and Analysis of System Architecture Using Rapide", IEEE Transactions on Software Engineering, 21(4):336-355, 1995

[45] Mens, T., “A Formal Foundation for Object-Oriented Software Evolution”, PhD. Dissertation, Vrije University, 1999

[46] MDA, http://www.omg.org/mda/ 
[47] Mills, H. D., 1971, “Top-Down Programming in Large Systems”, Debugging Techniques in Large Systems, Prentice-Hall, ISBN: 0138221227, 1971

[48] Myers, T., et al., "Seamlessly Integrating Software \& Hardware Modeling for Large-Scale Systems", 2nd International Workshop on Equation-Based Object-Oriented Languages and Tools, Paphos, Cyprus, July 2008

[49] Naumovich, G., Avrunin, G.S., Clarke, L.A., Osterweil, L.J., “Applying Static Analysis to Software Architecture", Proceedings of the 6th European Software Engineering Conference. Pp77-93, 1997

[50] Nejati, S., Sabetzadeh, M., et al., "Matching and Merging of Statecharts Specifications", 29th International Conference on Software Engineering, pp.54-64, 2007

[51] Nuseibeh, B., Easterbrook, S., "Requirement Engineering: a Roadmap", The Future of Software Engineering, Anthony Finkelstein (Ed.), pp.4-1, ACM Press 2000

[52] V.Phillips, (Raytheon Australia), "Implementing a Behavior Tree Analysis Tool Using Eclipse Development Frameworks", Australian Software Engineering Conference (ASWEC'08), Perth, March 2008

[53] Pilato, C, Collins-Sussman, B, et al. "Version Control With Subversion", ISBN 0596510330, O’Reilly Media, Inc., 2008

[54] Potts, C, "Requirements Model in Context", 3rd International Symposium on Requirements Engineering (RE'97), pp.102-104, 1997

[55] Rajlich, V., "Software Change and Evolution”, SOFSEM'99, LNCS 1725, pp.189-202, 1999

[56] Ramil, J. F., Lehman, M. M., "Metrics of Software Evolution as Effort Predictors - A Case Study", International Conference on software Maintenance. IEEE Computer Society Press, pp.163-172, 2000

[57] Royce, W., "Software Project Management, a Unified Framework", Addison Wesley Longman, Inc. ISBN 0201309580, 1998

[58] Saaltink, M., "The Z/EVES System", 19th International conference on the Z Formal Method (ZUM)”, Reading, UK, LNCS 1212, pp. 72-88, 1997

[59] Sabetzadeh, M., Easterbrook, S., "An Algebraic Framework for Merging Incomplete and Inconsistent Views", Proceedings of 13th IEEE International Conference on Requirements Engineering, pp.306318, 2005

[60] Schneider, S., "The B-Method - An Introduction", ISBN: 033379284X, Palgrave Editor, 2001

[61] Selonen, P.: "A Review of UML Model Comparison Techniques"; p.37-51 in: Proc. 5th Nordic Workshop on Model Driven Engineering, 27-29 August 2007, Ronneby, Sweden; Research report, U. Göteborg; 2007

[62] Shaw, M., DeLine, R., et al., “Abstractions for Software Architecture and Tools to Support Them”, IEEE Transactions on Software Engineering; 21(4)314-335, 1995

[63] Shlaer, S., Mellor, S.J., "Structured Development for Real-Time Systems", Vols. 1-3, Yourdon Press, 1985.

[64] Smith, C., Winter, K., Hayes, I., Dromey, R.G., Lindsay, P., Carrington, D., “An Environment for Building a System Out of its Requirements", Proceedings of the 19th IEEE International Conference on Automated Software Engineering, pp. 398-399, 2004

[65] Software Case Study of the Satellite System (Original Requirement), http://www.beworld.org/BE/resource/casestudy/satellite/scsoreq.pdf

[66] Software Change Case Study Site http://www.beworld.org/BE/resource/casestudy/satellite/

[67] Sommerville, I., "Software Engineering", $9^{\text {th }}$ Edition, Addison Wesley, ISBN:0137053460, 2011

[68] Swaina, R.K. et al, "Test Case Design Using Slicing of UML Interaction Diagram”, 2nd International Conference on Communication, Computing \&amp; Security, Pages 136-144, Volume 6, 2012,

[69] Swanson, E. B., "The Maintenance 'Ice Berg", Proceedings 2nd International Conference on Software Engineering. 10(10) pp. 1-14, 1972.

[70] Stafford, J. A., Wolf, A., L., "Architecture-Level Dependence Analysis for Software Systems", International Journal of software Engineering and Knowledge Engineering, Vol. 11 No. 4 pp. 431453,2001

[71] Sun, C., et al.: "Achieving convergence, causality-preservation, and intention-preservation in realtime cooperative editing systems", ACM Transactions on Computer-human Interaction, 5(1), pp. 63108, 1998

[72] Tracz, W., "DSSA (Domain-Specific Software Architecture): pedagogical example", ACM SIGSOFT Software Engineering Notes, Volume 20 , Issue 3, pp 49-62, July 1995

[73] Tuffley, D., Rout, T (2009), Applying Behavior Engineering To Process Modeling, in Proceedings of the 1st Improving Systems and Software Engineering Conference (ISSEC), National Convention Centre, Canberra, Australia, 10-12 August 2009.

[74] Trigg, R. H., and Weiser, M. “Textnet: A Network-Based Approach to Text Handling”, ACM Trans. Office Information Systems, Jan. pp.1-23, 1986

[75] Yau, S.S. Colofello, J. S. and MacGregor, T., "Ripple Effect Analysis of Software Maintenance", COMPSAC, pp. 60-65,1978 
[76] Wen, L., Dromey, R.G., “Architecture Normalization for Component-based Systems”, in Electronic Notes in Theoretical Computer Science, Elsevier BV, Radarweg 29, 1043 NX Amsterdam, Netherlands, vol.160, pp. 335-348, 2006

[77] Wen, L., Dromey, R.G., "From Requirements Change to Design Change: A Formal Path", Proceedings of the 2nd IEEE International Conference on Software Engineering and Formal Methods, pp. 104-113, 2004.

[78] Wen, L., Colvin, R., et al., “'Integrare', a Collaborative Environment for Behavior-Oriented Design”, Proceedings of the 4th International Conference on Cooperative Design, Visualization and Engineering (CDVE), 2007

[79] Wen, L., Kirk, D. , Dromey G., “A Tool to Visualize Behavior and Design Evolution”, Foundations of Software Engineering, 9th international workshop on Principles of software evolution: in conjunction with the 6th ESEC/FSE joint meeting, pp 114 - 115, 2007

[80] Wen, L., Dromey, R.G., "A Hierarchical Architecture for Modeling Complex Software Intensive Systems Using Behavior Trees", Proceedings of the 9th Asia-Pacific Complex Systems Conference, pp 292-299, 2009

[81] Wieringa, R. J., "Requirements Engineering: Frameworks for Understand”, ISBN: 0471958840, Wiley, 1996

[82] Winter, K., "Formalising Behavior Trees with CSP”, International Conference on integrated Formal Methods, IFM'04, pp. 148-167, 2004

[83] Zafar, S., Dromey, R. G., "Integrating Safety and Security Requirements into Design of an Embedded System”, Asia-Pacific Software Engineering Conference (APSEC'05), pp. 629-636, 2005

[84] Zhao, J., Yang, H., et al., "Change impact analysis to support architectural evolution", Journal of Software Maintenance and Evolution: Research and Practice; 14:317-333, 2002

[85] Zhao, J., "Change Impact Analysis for Aspect-Oriented Software Evolution”, International Workshop on Principles of Software Evolution, pp.108-112, 2002 